\title{
Emotional states and self-confidence independently fluctuate at different time scales
}

\author{
María da Fonseca ${ }^{1,2^{*}}$, Giovanni Maffei ${ }^{3}$, Rubén Moreno-Bote ${ }^{1,4,5}$, Alexandre Hyafil ${ }^{6}$
}

\author{
${ }^{1}$ Center for Brain and Cognition, Barcelona, Spain \\ ${ }^{2}$ Laboratory of Applied Artificial Intelligence, Computer Science Institute, School of Exact \\ and Natural Science, University of Buenos Aires, Argentina \\ ${ }^{3}$ Koa Health B.V., Barcelona, Spain \\ ${ }^{4}$ Department of Information and Communication Technologies, Universitat Pompeu Fabra, \\ Barcelona, Spain \\ ${ }^{5}$ Serra Hunter Fellow Programme, Universitat Pompeu Fabra, Barcelona, Spain \\ ${ }^{6}$ Centre de Recerca Matemàtica, Bellaterra, Spain \\ *e-mail: mariadafon@gmail.com
}

\begin{abstract}
Emotional states are an important ingredient of decision-making. Human beings are immersed into a sea of emotions where episodes of high mood alternate with episodes of low mood. While changes in mood are well characterized, little is known about how these fluctuations interact with metacognition, and in particular with confidence about our decisions. Here, we evaluate how implicit measurements of confidence are related with mood states of human participants through two online longitudinal experiments involving mood self-reports and visual discrimination decision-making tasks. Self-confidence was assessed on each session by monitoring the proportion of opt-out trials when an opt-out option was available, as well as the mean reaction times on standard correct trials. We first report a strong coupling between the mood, stress, food enjoyment and quality of sleep reported by participants in the same session. Second, we confirmed that the proportion of opt-out responses as well as reaction times in non-opt-out trials provided reliable indices of self-confidence in each session. We introduce a normative measure of overconfidence based on the pattern of opt-out selection and the signal-detection-theory framework. Finally and crucially, we found that mood, sleep quality, food enjoyment and stress level are not consistently coupled with self-confidence, but rather they fluctuate at different time scales: emotional states display faster fluctuations (over one day or half-a-day) than self-confidence level (two-and-a-half days). Therefore, our findings suggest that emotional states and confidence in decision making spontaneously fluctuate in an independent manner in the healthy adult population.
\end{abstract}




\section{Highlights}

- Longitudinal study tracking affective states and decision uncertainty of subjects for a period of 10 consecutive days in everyday life settings.

- Self-reported emotional states significantly correlate with each other.

- The proportion of opt-out responses (allowing skipping of the decision) and reaction time in non-optout correct trials reflect decision uncertainty in two discrimination tasks.

- There is no significant correlation between daily fluctuations of emotional states and self-confidence markers.

- Emotional states and self-confidence fluctuate at different time scales.

\section{Introduction}

Emotions and cognition have long been known to interact (Damasio, 2008). In particular, emotional states modulate decision-making and especially metacognition, that is the monitoring of one's own thought process and performance (Eldar et al., 2016; Vinckier et al., 2018). For example, inducing a transient state of sadness or anxiety can shift a subject's willingness to perform a risky decision (Raghunathan \& Pham, 1999) or boost the accuracy of confidence judgments (Massoni, 2014). On a longer time scale, personality traits also affect metacognitive judgments $(\mathrm{H}$. Xu, 2020), while metacognitive impairments and emotional dysregulation are associated in various psychiatric disorders (Rouault et al., 2018). In particular, these disorders lead to an unbalanced sense of self-confidence or simply confidence (Hoven et al., 2019), which refers to our capacity to perform and report robust evaluations of our decisions, and use these evaluations to control our decision-making (Yeung \& Summerfield, 2012). Confidence allows us to know when a decision is too risky to take and to revert decisions based on more recent and more compelling evidence. For example, confidence judgments is impaired in depressed patients (Fu et al., 2005), who consistently underestimate their performance level, which could have a major impact in preventing these patients from taking the appropriate decisions to improve their condition. On the converse, schizophrenic and psychotic patients usually display strong overconfidence (Averbeck et al., 2011; Jardri \& Denève, 2013; Moutoussis et al., 2011; Rubio et al., 2011).

Our understanding of the association between affects and metacognition is limited by the gap in the time-scale between short-lived experimentally-induced emotional states and long-lasting states such as affective disorders. The link between spontaneous daily fluctuations in mood and metacognition in ecologically valid settings remains poorly understood. Only a handful of studies have shown that positive events such as good weather or a sports win lead to more risk-seeking behavior, probably through their impact on mood (Bassi et al., 2013; Edmans et al., 2007; Otto et al., 2016). The impact of mood on confidence remains however unknown. In fact, whether confidence fluctuates and at which time scale has to our knowledge never been studied, beyond the mere introspective experience that we sometimes feel more confident. Popular sayings seem to take such associations between mood state and alterations of metacognition for granted: "Do not promise when you are happy, do not decide when you are sad". Testing such association could be performed by tracking spontaneous emotional states and cognition simultaneously 
through longitudinal studies. Online tools now make longitudinal studies with many behavioral sessions in a cohort of participants much more affordable to the experimenter (Gillan \& Rutledge, 2021) and offer the possibility to study cognitive processes in ecological settings precluded to lab experiments. This is of particular importance for the translation of scientific insights to clinical applications such as the diagnostics of affective disorders or the development of predictive models for crisis prevention using digital phenotyping techniques, i.e. making inference of emotions from patient's digital data "in the wild" (Dagum, 2018; Jones et al., 2021; Taylor et al., 2017). To our knowledge, only one study so far has investigated the coupling between fluctuations of mood and cognition (more specifically, value-based decision-making patterns) through longitudinal studies in a group of healthy adult participants learning (Eldar et al., 2018). But it remains unknown whether daily mood fluctuations interact with metacognitive states such as self-confidence.

Based on the ideas exposed above, we hypothesize an association between daily fluctuations in mood and self-confidence. To address the above hypothesis, we developed two online longitudinal experiments where adult volunteers reported their mood, sleep quality, food enjoyment and stress level, and performed one of two simple visual discrimination task twice per day during ten consecutive days. We inferred the level of self-confidence by tracking how often subjects chose an opt-out option, available in a fraction of the visual task trials, which allowed avoiding to report the perceived stimulus. If mood and confidence fluctuations are indeed linked, we expect the participants to opt out less often during high mood episodes, whereas during low mood episodes they recur more to opting out. We also used median reaction times in a session as another indicator of the participant's confidence (Moreno-Bote, 2010; Urai et al., 2017; Vickers, 1979, 2014). Our results showed that both proxies of self-confidence are reliable markers of the session confidence level. Self-reported emotional states were highly correlated with each other. In contrast to our original hypothesis, we found that spontaneous fluctuations in mood and confidence were not coupled, but evolved on different time scales. Our results challenge the idea that fluctuations of mood and confidence are intrinsically coupled in the healthy adult population.

\section{Methods}

\section{General structure of the paradigm}

All participants were invited to complete twenty sessions in ten consecutive days, that is, two sessions per day, one in the morning (8-12 AM) and the other in the afternoon (4-8 PM), starting on a Thursday morning and ending on a Saturday afternoon (Figure 1). The sessions were targeted to take about 10 minutes. In total 27 participants ( 20 female, 6 male, 1 other) and 23 participants (18 female, 4 male, 1 preferred not to answer) were recruited for the Numerosity (NT) and the Orientation task (OT), respectively (10 of them participated in both), mainly among students from the Pompeu Fabra University. The median age was 25 (minimum 19, maximum 42) for the NT and 23 (minimum 20, maximum 34) for the OT. We accepted all healthy Spanish-speaking adults with normal or corrected to normal vision. One participant (in each task) reported a neurological or psychological/psychiatric disorder diagnosed by a professional, 2 (1) preferred not to report about a possible disorder in the NT (OT). We obtained online confirmation of informed consent to the conditions and the payment modalities of the task. Irrespective of their performance, they were paid $2.5 €$ per 
session ( $5 €$ per day) and $40 €$ bonus for having completed all the sessions properly. Additionally, they had the chance to obtain a bonus payment which was determined by their final score in the session; the two highest scoring participants of each session received a $4 €$ bonus. The session score was computed as the sum of the score of each trial: 3 (0) points for correct (incorrect) answers; 2 points for deterministic opt-out election; 3 or 0 points, randomly assigned, for stochastic opt-out choice (see below). The score and bonus schemes were explained to the subject by written task instructions. We informed participants of the bonus money they received after the end of the whole experiment, to prevent feedback biases and maintain their level of motivation.

Participants were allowed to take a break between stages. A fraction number layed out in the instructions title marked the current stage of the experiment, and the approximate time duration of the current stage was also displayed. We excluded data from three sessions of one participant in the NT, and two sessions (from two different participants) in the OT, where median reaction time for some difficulty levels (see Methods - Stimuli \& responses) exceeded 2 seconds, and data from three incomplete sessions (from two different participants) in the OT. The study was approved by the Ethics Committee of the Department (CIREP approval \#121).

\section{Stimuli \& responses}

The participants performed the experiment via a browser on their personal computers through the Jatos online platform (Lange et al., 2015). The experiment was custom-made in JavaScript and data were collected on an institutional server managed by the Jatos team (Lange et al., 2015). Prior to the first session, we provided to each participant a personal link to be used once per session and an online presentation with detailed instructions and a few examples of the decision-making task. The first screen in each session instructed the participants to make sure they had the right environmental conditions: be in an indoor room, turn the brightness screen up to maximum, avoid any light source behind the screen, and to place themselves at $60 \mathrm{~cm}$ from the center of screen.

A demographic questionnaire was displayed in the first session with questions about participant's age, gender, country of residence, education level, use of lens, whether they were diagnosed with a neurological or psychological/psychiatric disorder and/or took medication. The reply options were predetermined alternatives to scroll and select.

In each session (Fig. 1), we presented a questionnaire with $3 / 4$ questions about their quality of life. In the morning session, questions were (in Spanish): 'How have you felt this morning?' (mood), 'How did you sleep last night?' (sleep), 'How did you enjoy your last meal/snack?' (food), and 'How did you feel about your personal and working problems this morning?' (stress). In the afternoon session, questions were the same mood, food and stress inquiries asked in the morning session, with the word 'afternoon' instead of 'morning', while the sleep question was skipped. To answer each of the 4 (morning) or 3 (afternoon) self-reports, participants placed a cursor with the mouse along a horizontal continuous-scale with sad and smiling emoji faces at the ends (see Supplementary Figure 1). The cursors for the different self-reports were initially placed at the middle of the corresponding bar (all presented on the same screen), and responses could not be validated until all cursors were moved, preventing skipping the report. The response was linearly mapped onto an interval between $[0,1]$. Because the sad (smiling) emoji face was placed at the left (right) extreme, the corresponding quantity for the stress-report was inverted as (1 - cursor-position) so that a high value indicates high level of stress. 
After the questionnaire, we presented a two-alternative (left/right) visual discrimination task. The participants answered after a $300 \mathrm{~ms}$ presentation of the stimulus by pressing the left/right arrow of the keyboard. If the participants chose the correct answer, they received 3 points. An incorrect choice did not yield any point. In a fraction of trials, the participants had the possibility to select an opt-out option reflected by a third response option represented by the up arrow of the keyboard. By choosing this option, the subject skipped the decision and passed to the next trial, but obtained a certain or stochastic reward, explained in what follows. There were two stages in each session which differed in the reward scheme for opt-out responses, and were composed of the same number of trials (120 trials for NT and 90 trials for OT). In both stages, two-thirds of the trials corresponded to opt-out trials (80 trials for NT, 60 for OT), while the remaining third of trials corresponded to non-optout trials where the option was not presented (40 trials for NT, 30 for OT). In the first stage, the Deterministic Opt-out (DO) stage, the opt-out option (in opt-out trials) was represented by a question mark icon displayed during the response window (see Figure 1) and returned a fixed number of 2 points. In the second stage, the Stochastic Opt-out (SO) stage, the opt-out option was represented by a dice icon and the number of points were chosen randomly: in $80 \%$ of the trials the opt-out option returned 3 points, while the other $20 \%$ did not return points. After selecting the opt-out option in the SO stage, participants received a feedback coin indicating the amount of points they received. This contrasted with the non-optout response trials and opt-out responses in the DO stage where no feedback was provided. Written instructions were displayed at the beginning of each stage informing the score scheme in place for the stage.

Before each stimulus presentation, a cue consisting of either a cross, a question mark or a dice was presented for $300 \mathrm{~ms}$, indicating that the trial would be a non-optout, DO or SO trial, respectively. In the NT (see Figure 1 ), two empty circles (radius $=15 \%$ of screen width) were presented on each side of a central cross (size $=20 \times 20$ pixels) for $300 \mathrm{~ms}$. Then, white dots (radius $=20$ pixels each) appeared within each of the circles for $300 \mathrm{~ms}$ (adequate grid spacing was introduced to prevent the circles from overlapping) (Fleming et al., 2016). One of the circles always contained 50 dots and the other a larger number of dots. The difficulty of the trial was manipulated by controlling the number of dots in the larger set of dots, which could be either $52,56,60$ or 64 . Participants were instructed to maintain fixation on the central small cross placed between the two circles and report whether the left or right circle included more dots. In the OT, the stimulus consisted of a noisy Gabor patch (radius $=216$ pix., period $=72$ pix., phase $=0$; envelope $=150$ pix., middle contrast, middle average luminance) tilted either to the left $\left(-45^{\circ}\right)$ or right $\left(45^{\circ}\right)$. The stimulus was presented at fixed contrast in the center of the screen on a middle gray background. After the stimulus presentation, the Gabor image disappeared for a short delay of $300 \mathrm{~ms}$ followed by presentation of the different response options. The difficulty of the trial was controlled by manipulating the level of noise, from 0 (noiseless Gabor patch) to 1 (completely noisy Gabor patch), following (Wyart et al., 2012). We used three levels of difficulty corresponding to levels of noise of $\left\{n_{s}-0.08, n_{s}, n_{s}+0.08\right\}$ where $n_{s}$ is a session-adjusted noise level defined using an adaptive procedure (see below).

In both tasks, following stimulus presentation, the response options appeared on the screen, in accordance with the cue, without time limitation to answer. The options disappeared immediately after the participant's response, and the following trial started $300 \mathrm{~ms}$ after the button press. In a small fraction of trials, the actual stimulus presentation appeared longer than 300 ms due to small timing inaccuracies in the Internet browser. We excluded all trials where the stimulus display exceeded $350 \mathrm{~ms}$. 
Training and adaptive procedure. In the NT, on every session, a practice stage of 10 trials was performed before the DO and SO stages. The stimulus difficulty of the practice stage followed a staircase procedure, starting with 20 points of difference between the two circles and decreasing (increasing) by 3 points after correct (incorrects) replies. During this practice stage, performance feedback was provided after each trial, consisting of the image of a coin with 3 points for correct and 0 point for incorrect trials.

In the OT, a practice block of 10 trials was performed in the first participant session only. Noise level was set to $30 \%$, and feedback was provided as for practice trials in the NT. On every session, following task instructions, participants performed a block of 60 non-optout trials where the level of stimulus noise was adjusted following an adaptive staircase to adjust the participant average accuracy on the stimulus to reach $66 \%$. The value of the noise at the end of this block defined $n_{s}$, i.e. the level of noise for the middle difficulty trials in the rest of the session. Stimuli were picked up from a library of pre-selected images generated with noise levels sampled according to their energy along the diagonal directions (Wyart et al., 2012).

\section{Autocorrelation analysis}

We computed the autocorrelation for report variables (mood, stress, etc.) and psychometric variables extracted from the decision making task in each session (proportion of opt-out responses, overall performance in non-optout trials, median reaction time in non-optout correct trials, etc.). For each variable, we first calculated the autocorrelation (AC) as the Pearson correlation coefficient between the temporal series (consisting of one data point of each variable per session) and the same series shifted by $k$ sessions (or $k$ days for sleep). For the psychometric variables, we subtracted the mean across participants to the variable in order to remove possible biases due to learning effects consistent across subjects. The AC of short temporal series is biased negatively (Marriott \& Pope, 1954). We removed this negative bias by subtracting from the $A C$ vector the average $A C$ vector from 100 randomly shuffled versions of the corresponding temporal series. Finally, for each variable, we computed the mean and standard error of the mean (s.e.m.) of the bias-corrected AC across participants. We corrected the p-values for comparison at different lags by using the following iterative procedure: we first tested significance at lag 1 ; if the $p$-value was larger than 0.05 , then autocorrelation coefficients were judged non-significant at all lags; if the p-value was lower than 0,05 , the coefficient was judged significant at lag 1 and we moved on to assessing $p$-value at lag 2; and iteratively until we found the first lag where the associated $\mathrm{p}$-value was larger than 0.05 .

\section{Cross-correlation analysis}

We computed the cross-correlation between report variables (mood, stress, etc.) and psychometric variables extracted from the decision making task in each session. For each variable, we first calculated the cross-correlation (CC) as the Pearson correlation coefficient between the temporal series consisting of one data point of one of the report variables per session and the temporal series shifted by $k$ sessions (only morning sessions in CC with sleep) of one of the psychometric variables. We subtracted the mean across participants to the variable in order to remove possible biases due to learning effects consistent across subjects, and removed the negative bias present in short temporal series following the same procedure for the autocorrelation computation. Finally, for each pair of variables, we computed the mean and standard error of the mean (s.e.m.) of the bias-corrected CC across 
participant, and corrected the p-values for comparison at different lags by using the iterative procedure used for the autocorrelation for both negative and positive lags.

\section{Difficulty-adjusted across-session correlations}

We refined the across-session correlations of psychometric variables, and in particular the proxies of self-confidence (reaction time in correct non-optout trials and the proportion of opt-out) whose within-session variance is inflated by the different difficulty levels. To do so, we first computed the mean value for each variable (median for RT) separately for each difficulty level, session and subject, resulting in a total of $n=n_{\text {sessions }}(20) \times n_{\text {difficulties }}$ (4 for NT and 3 for OT) points per participant. We then corrected each point per difficulty level by subtracting the mean value of the variable across sessions for that difficulty level, i.e. we centered the data point. Finally, these difficulty-residuals were entered into a linear mixed model (with statsmodels Python library), with the residual RT as the dependent variable, the residual DO or SO as fixed effect and session as a random effect. The difficulty-adjusted across-session correlation corresponds to the weight of the fixed effect in the linear mixed model.

\section{Psychometric curves}

We fitted the responses of each participant in each session by a psychometric curve, separately in non-optout, DO and SO trials. The psychometric curve captures the proportion for each response type (left, right, opt-out) as a function of the signed stimulus evidence (positive stimulus indicates evidence in favor of the right option). In the NT, the signed stimulus evidence was defined as the difference in the number of points between the right and left circles. In the OT, the signed stimulus evidence was defined as the difference in motion energy between 45 and -45 degrees. The psychometric curve for non-optout trials was computed by grouping non-optout trials of DO and SO stages from the same session.

Following Signal Detection Theory (SDT), the psychometric curve is determined by the level of perceptual and decision noise $\sigma^{2}$ and the decision boundary $H$. When we present a stimulus strength $e$, the participant observes $\hat{e}=e+\eta$, where $\eta \sim N\left(0, \sigma^{2}\right)$, with $N$ the gaussian distribution, and the participants categorizes the stimulus to the right category if $\hat{e}>$ $H$, and to the left if $\hat{e}<H$. Thus, the probability of answering rightwards can be written as

$p($ rightward $\mid e)=\int_{H}^{+\infty}(e+\eta) d \eta=\Phi\left(\frac{e-H}{\sigma}\right)$,

where $\Phi$ is the standard normal cumulative density function. We estimated the internal noise and the decision boundary by fitting the psychometric curve with the probit regression using the statsmodels Python library (Fig. $5 \mathrm{~A}$ ).

In opt-out trials, participants could select between 3 options: L (leftward), R (rightward) and O (opt-out). Following SDT, we postulate that participants apply two decision boundaries in the perceptual space: $H_{L}$ between the leftward and opt-out responses, and $H_{R}$ between the opt-out and rightwards responses (García-Pérez \& Alcalá-Quintana, 2017). This gives rise to the following equations for the proportion of the leftwards, rightwards and opt-out responses as a function of stimulus evidence $p_{L}(e)=1-\Phi\left(\frac{e-H_{L}}{\sigma}\right), \quad p_{R}(e)=\Phi\left(\frac{e-H_{R}}{\sigma}\right)$ and $p_{o}(e)=1-p_{L}(e)-p_{R}(e)$. This model corresponds to an ordered probit regression. We estimated $H_{L}, H_{R}$ and $\sigma$ from each session data through the maximum likelihood estimation 
method. In the few sessions where the participant did not opt out for a single trial, we instead fitted the psychometric curve as in the non-optout trials.

\section{Optimal decision boundary in opt-out trials}

If the participants know their internal perceptual noise level, then they can adjust the boundaries in the opt-out conditions in order to maximize the expected number of points associated with the response (Barrett et al., 2013). In deterministic opt-out trials, given the point scheme, the expected number of points of the opt-out, left and right responses is respectively 2 points, $3 p(e<0 \mid \hat{e})$ and $3 p(e>0 \mid \hat{e})$, respectively, where the conditional probabilities refer to the probability that the true stimulus evidence $e$ is positive or negative given the observed evidence ê. According to the optimal observer model, the rightward decision boundary $\left(H_{R}\right)$ should be set as the observed evidence $\hat{e}$ where the expected number of points for rightward and opt-out responses are equated, i.e. where $p(e>0 \mid \hat{e})=2 / 3$. In other words, the optimal observer should select the rightward response only if its expected accuracy is above $2 / 3$; otherwise, it is more convenient to opt-out and collect 2 points. Thus, the boundary $H_{R}$ should be placed such that $p\left(e>0 \mid \hat{\mathrm{e}}=H_{R}^{\text {opt }}\right)=2 / 3$, while the boundary $H_{L}$ should be placed where $p\left(e>0 \mid \mathrm{e}=H_{L}^{o p t}\right)=1 / 3$.

The Bayes rule gives us that $p(e \mid \hat{\mathrm{e}}) \propto p(e) p(\hat{\mathrm{e}} \mid e)$, i.e. the posterior over each stimulus strength depends both on the prior evidence for the stimulus strength and the likelihood of the stimulus strength given the observed evidence. Assuming that the bias displayed by participants in the trials without the opt-out option is a perceptual bias $H$, then $p(\hat{\mathrm{e}} \mid e)=N\left(\hat{\mathrm{e}} \mid e-H, \sigma^{2}\right)$, where the variance of the sensory noise $\sigma^{2}$ can be estimated from the resulting fit of the non-optout psychometric curves and e corresponds to the signed stimulus strength corresponding to the presented stimulus. Here we assume that the prior over the stimulus strength is a gaussian centered on 0, i.e. $p(e)=N\left(e \mid 0, \varepsilon^{2}\right)$, where $\varepsilon$ is the standard deviation of $e$. While the true prior distribution is over a set of $6 / 8$ discrete values, it is unlikely that participants notice such a discrete distribution from a noisy set of observations. Rather we assume that they use such gaussian form with the same standard deviation as the standard deviation of the true distribution (notice that the gaussian form is the maximum-entropy distribution, i.e. the less informative distribution when the mean and standard deviation are known). We can then further develop:

$p(e \mid \hat{\mathrm{e}}) \propto p(e) p(\hat{\mathrm{e}} \mid e)=N\left(e ; 0, \varepsilon^{2}\right) N\left(\hat{\mathrm{e}} ; e+H, \sigma^{2}\right) \propto N\left(e ; \mu, v^{2}\right)$, with $\mu=(\hat{\mathrm{e}}-H) \varepsilon^{2} /\left(\varepsilon^{2}+\sigma^{2}\right)$ and $v^{2}=\sigma^{2} \varepsilon^{2} /\left(\varepsilon^{2}+\sigma^{2}\right)$.

Thus $p(e>a \mid \hat{\mathrm{e}})=\int_{e>a} p(e \mid \hat{\mathrm{e}}) d e \propto \int_{e>a} N\left(e ; \mu, v^{2}\right) d e=\Phi\left(\frac{\mu-a}{v}\right)$. Using the limit $a \rightarrow-\infty$ where $p(e>a \mid \hat{e}) \rightarrow 1$, we see that the coefficient of proportionality is 1 . Hence:

$p(e>0 \mid \hat{\mathrm{e}})=\Phi\left(\frac{\mu}{v}\right)$.

The implicit equation $p\left(e>0 \mid \hat{\mathrm{e}}=H_{L}{ }^{\text {opt }}\right)=1 / 3$ can now be solved:

$\Phi\left(\frac{\mu}{v}\right)=1 / 3 \Rightarrow \mu=v \Phi^{-1}(1 / 3) \Rightarrow$

$H_{L}^{o p t}=H+\left(\varepsilon^{2}+\sigma^{2}\right) / \varepsilon^{2} v \Phi^{-1}(1 / 3)=H+\sigma \sqrt{1+(\sigma / \varepsilon)^{2}} \Phi^{-1}(1 / 3)$. 
Similarly for the optimal right boundary:

$H_{R}^{o p t}=H+\left(\varepsilon^{2}+\sigma^{2}\right) / \varepsilon^{2} v \Phi^{-1}(2 / 3)$.

In the stochastic opt-out trials, the expected number of points for the opt-out response is $0.8^{*} 3=2.4$ (as the 3 point reward is obtained in $80 \%$ of trials), so the optimal boundaries should be computed accordingly.

Overconfidence (OC). We defined the overconfidence associated with a participant's session as $O C=\frac{\left|H_{L}^{\text {opt }}-H_{R}^{\text {opt }}{ }_{R}\right|-\left|H_{L}-H_{R}\right|}{\left|H^{\text {opt }}-H^{\text {opt }}{ }_{R}\right|+\left|H_{L}-H_{R}\right|}$. The value is bounded between -1 and 1 : it is positive when the participant opted out less than according to the optimal strategy, reflecting overconfidence, and negative otherwise, reflecting underconfidence. Notice that the distance between optimal boundaries is independent of the perceptual bias $H$ but scales with the standard deviation of the perceptual noise $\sigma$ :

$$
\left|H_{L}^{o p t}-H_{R}^{o p t}\right|=2 \sigma \sqrt{1+(\sigma / \varepsilon)^{2}} \Phi^{-1}(2 / 3) \text {. }
$$

Risk aversion (RA). We defined risk aversion as the difference between the proportion of opt-out responses in the stochastic (SO) and deterministic (DO) stages of each session (bounded between -1 and 1).

Bayesian linear mixed models (BLMM). We defined bayesian linear mixed models using participant as random variable and a confidence proxy (either the average proportion of optouts or the median reaction time in correct NO trials in the session) as the dependent variable. The bayesian mixed model formula was confidence_proxy $\sim 1+1$ |participant + report + report|participant, where report corresponded to one of the four self reports. In other words, random factors include both the intercept and sensitivity to the report. In other words, this model could account for a subject-specific dependence of the confidence proxy on the report. We also included a control model where the dependence on the proxy was absent, i.e. confidence_proxy $\sim 1+1$ participant. Bayesian mixed models were fitted using the bambi package in Python (Yarkoni \& Westfall, 2016) with Gaussian priors (with mean equal to the grand average of the confidence proxy for the fixed intercept; mean equal to zero for all other parameters; and half-student $\mathrm{T}$ and half normal distributions for the standard deviation in fixed and random factor, respectively). We performed model selection using two different metrics: the Watanabe-Akaike Information Criterion (WAIC) and the LOO (leave-one-out) cross-validation criterion (using the arviz package in Python).

\section{Results}

In two longitudinal online experiments, participants reported their emotional states and performed a decision-making task twice a day, once in the morning and once in the afternoon, during 10 consecutive days, for a total of 20 sessions per participant (see Fig. 1). In each session, participants started by answering 3 (morning sessions) or 4 (afternoon) questions about their mood, the quality of their sleep, their food enjoyment and their stress level. After this self-report questionnaire, they performed one of two two-alternative forced choice decision-making tasks: one cohort of subjects ( $n=27$ participants) performed a numerosity task (NT) where they reported which of two simultaneously presented circles 
contained more dots (Fleming et al., 2016), and the other cohort ( $n=23$ participants) performed an orientation task (OT) where they reported whether a noisy titled Gabor patch was titled to the left or right (Wyart et al., 2012); see Methods. We controlled the difficulty of each trial by manipulating the difference between the number of dots in the circles (NT) or the difference in stimulus energy between rightward and leftward orientation of the noisy Gabor patches following (OT). In a fraction of the trials, participants were offered an option to opt out and skip the decision, which resulted in a certain or stochastic number of points. Participants were encouraged to use the opt-out option when they were uncertain of the stimulus category in order to maximize the collection of points over the session. Three points were achieved with a correct answer and zero points for an incorrect one. The opt-out option, when available, ensured a fixed amount of two points in the first stage of the session (Deterministic Opt-out, DO) or 3 points with probability $80 \%$ and 0 points with probability $20 \%$ in the second stage of the session (Stochastic Opt-out, SO). We hypothesized that the proportion of opt-out responses in the session would represent a robust index of the current level of self-confidence of the subject (Grimaldi et al., 2015). To determine whether the proportion of opt-outs is inflated by a risk-averse strategy, we introduced the SO task in the second stage in each session, where the levels of risk between the opt-out and non-optout strategies are similar.

We used another implicit measurement of the level of participant confidence on each session: the reaction time in trials without opt-out option (non-optout trial), which is known to be larger for uncertain decisions (Moreno-Bote, 2010; Urai et al., 2017; Vickers, 1979). We avoided explicit measures such as reporting decision confidence on a scale for each trial (Martino et al., 2013; Schustek et al., 2019) in order to prevent the possible direct influence of the emotional states over explicit metacognitive assessments. As introduced above, the SO stage allowed disentangling the effects of self-confidence and risk aversion in the selection of the opt-out options (Dienes \& Seth, 2010). We used the difference between the proportion of opt-out responses in the $\mathrm{DO}$ and $\mathrm{SO}$ stages in a single session as a direct index of risk aversion.

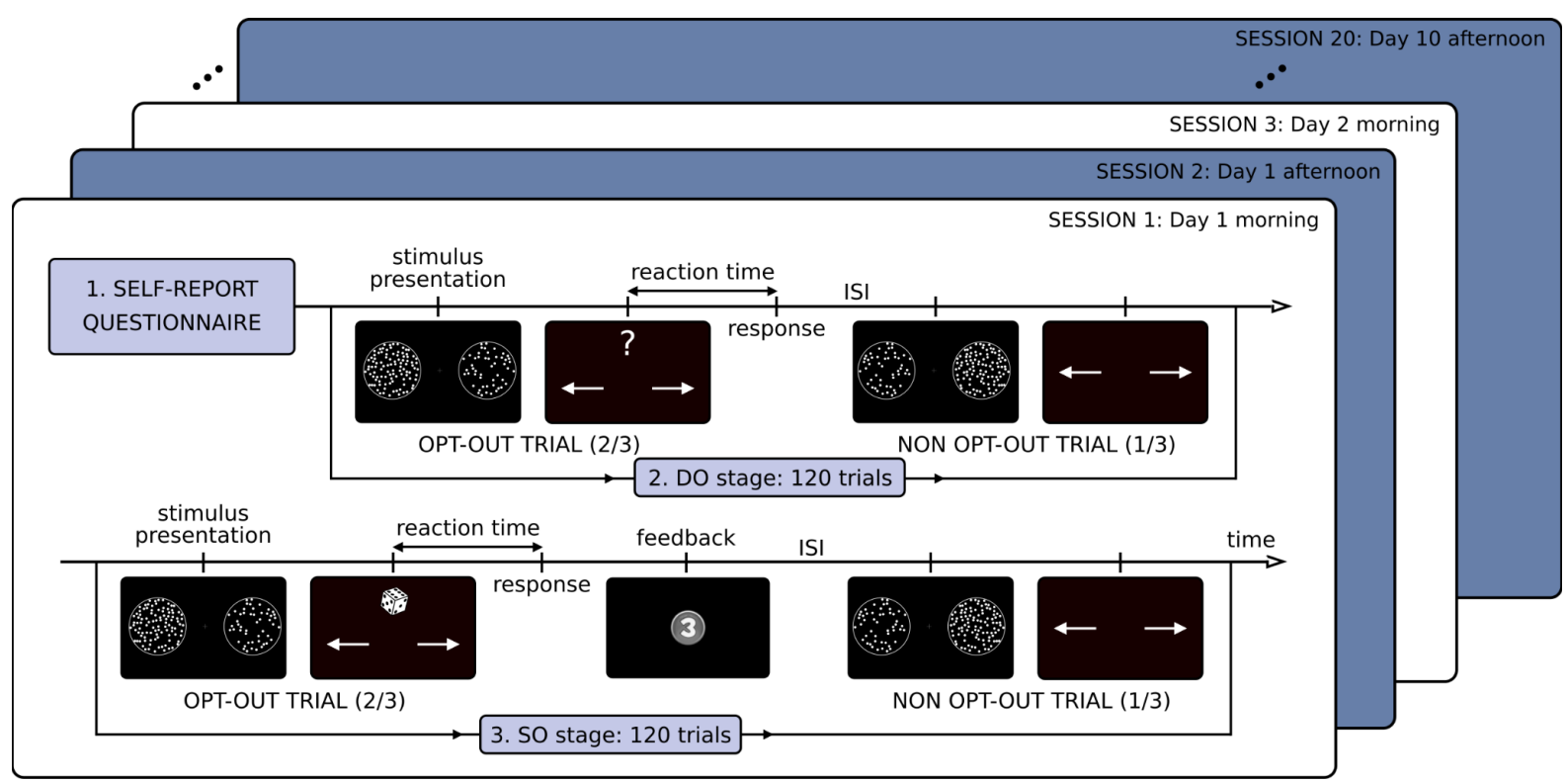

Figure 1. Experimental paradigm. The online experiment lasts 10 days with two sessions per day of approx. 10 minutes: morning (8-12 am) and afternoon session (4-8 pm). Each 
session starts with 3 or 4 questions about the participant's mood, the quality of their sleep, their enjoyment of the food, and their stress level. After the questionnaire, the participant completed a two-alternative forced-choice perceptual task. In two-thirds of the trials, they could select a third opt-out option ('?' symbol on the top left trial) which allowed skipping the decision. Participants were instructed to select the opt-out option when they were unsure of the stimulus category in order to maximize their cumulative score on the session. Two stages of trials in each session differed in contingencies of the opt-out option. In the first block called 'Deterministic Opt-out' (DO stage; top row), the opt-out option returns a fixed number of 2 points. In the second block called 'Stochastic Opt-out' (SO stage; bottom row), the opt-out option yields $80 \%$ of chance of obtaining returns 3 points (like in the example), while the other $20 \%$ do not return any point (in contrast to all other options, the number of points obtained was provided after choosing the stochastic opt-out option). On each trial task, a cue is presented prior to the stimulus, indicating the available options (an $\mathrm{X}$ for non-optout trials, a question mark for DO and a dice for SO).

\section{Daily fluctuations in self-reported emotional states covary}

The average values over participants of the self-report variables (mood, quality of sleep, enjoyment of food, stress) per session are illustrated in Figure 2A. They appeared mostly stable across days, with a significant difference between the mean across weekend and week sessions for mood (two-paired t-test across NT and OT participants: $t=2.1, p=0.04$ ), stress $\left(t=-4.02, p<10^{-3}\right)$ and food $\left(t=3.8, p<10^{-3}\right)$ reports. The difference was not significant for the sleep report $(t=1.1, p=0.3)$. This result indicates that, on average, participants were in a better mood, less stressed and ate better during weekend sessions compared with week sessions (Larsen \& Kasimatis, 1990; Stone et al., 2012). At the individual level, these variables fluctuated substantially per session. We found that mood, food and stress self-reports presented a significant positive autocorrelation at lag 1 (i.e., around 4-16 hours difference between the two reports) in both tasks (two-tailed t-test on 1-lag correlation values across subjects, $p<0.05$ for each variable in both experiments; Figure $2 \mathrm{~B}$ ). Looking in greater detail at the 1-session autocorrelation, we found a tendency for the auto-correlation to be weaker between reports on one afternoon and reports on the morning after than between reports from the same day's morning and afternoon (Supplementary Table 1), suggesting that mood states largely did not carry over from one day to another. For the food self-reports, the autocorrelation stayed significant at lag 2 (i.e. 24 hours difference between the two reports). This suggests that these markers fluctuate at the scale of one or half a day: slower fluctuations were not observed. Furthermore, we found strong correlations between all self-reported emotional states (Figure 2C): mood, food and sleep indicators were positively correlated, and negatively correlated with the stress level, as expected (all p-values remained significant after Bonferroni correction). This result indicates that, on average, when the participants slept and ate better, they also experienced a better mood and were less stressed. Remarkably, the whole pattern of correlations between reports was very similar in both experiments (Figure $2 \mathrm{C}$ ). Finally, we found significant correlations at the individual level in a large proportion of participants (Figure 2D, E). Overall, these results suggest a strong and reliable association between the daily fluctuations in mood, quality of sleep, stress and enjoyment of food. 
A
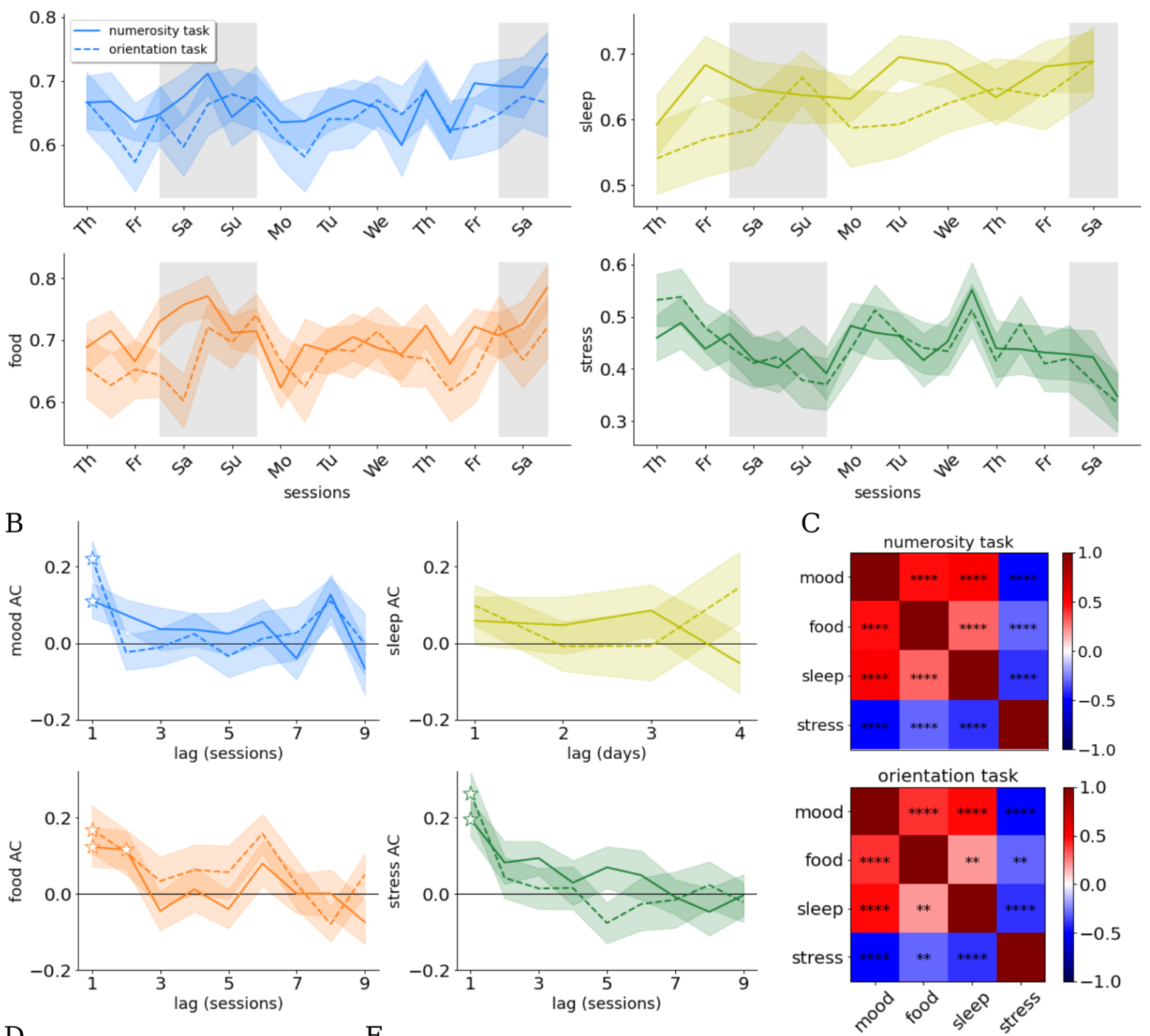

$\mathrm{D}$

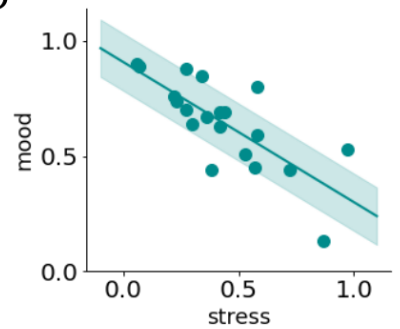

$\mathrm{E}$

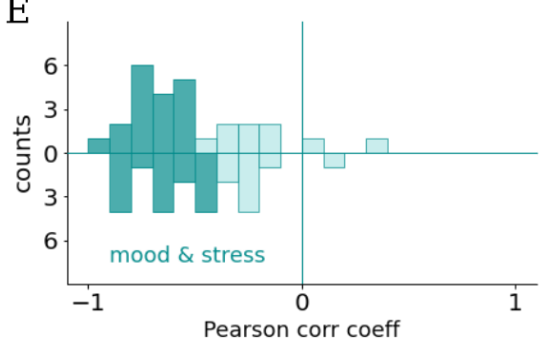

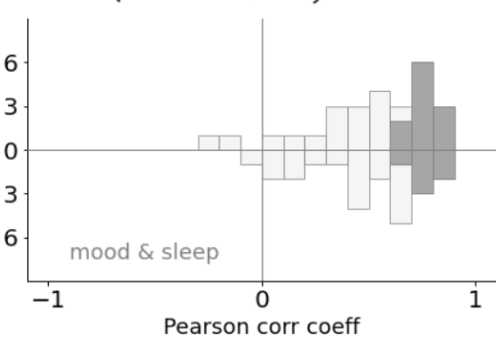

Figure 2. Session-to-session fluctuations in quality of life reports. A. Mean values per session for mood, food, stress and sleep self-reports across participants in the numerosity task group (full lines; $n=27$ ) and orientation task group (dashed line; $n=23$ ). Coloured shaded areas indicate standard error of the mean (s.e.m.). Gray shaded areas indicate weekend sessions. B. Autocorrelation coefficients in self-reports (star: t-test across participants, $p<$ 0.05 after correction for multiple lags, see Methods). C. Average correlation heat map of the self-report variables across participants ${ }^{* *} p<0.01$; $\left.{ }^{* * * *} p<0.0001\right)$. D. Mood vs. stress reports for one exemplar participant. Each dot represents a session. The line represents a linear fit while the shaded area represents the standard error of the estimated slope. E. 
Distribution of Pearson correlation coefficients between mood and stress (mood and sleep) in the left (right) panel across subjects. Filled bars indicate subjects with significant correlation at individual level ( $p<0.05$, uncorrected). Upright and inverted axes correspond to the numerosity and orientation task groups, respectively.

\section{Opt-out selection reflects the session-specific level of self-confidence.}

Next, we analyzed behavioral data in the numerosity task and assessed whether the proportion of opt-out options and reaction times constituted solid markers of choice confidence. As expected, the overall accuracy level dropped for increasing stimulus difficulty for non-optout, deterministic opt-out and stochastic opt-out trials (Figure 3A, top). Interestingly, accuracy was higher in trials where the opt-out option (either stochastic or deterministic) was presented but finally discarded by the participant than in trials where it was not presented. This was confirmed in a factorial 2-way ANOVA with difficulty and opt-out condition (non-optout, stochastic opt-out, deterministic opt-out) as factors: we found a main effect of opt-out condition $\left(F_{\text {condition }}=18.3, p<10^{-7}\right)$ as well as difficulty $\left(F_{\text {difficulty }}=451.4, p<10^{-113}\right)$, with no significant interaction between opt-out condition and difficulty $(F=0.6, p>0.7)$. Post-hoc t-tests showed that accuracy was lower in non-optout trials compared to deterministic ( $t=-5.1, \quad p<10^{-4}$ corrected) or stochastic opt-out trials $\left(t=-9.2, p<10^{-8}\right.$ corrected). This suggests that subjects were able to estimate on a trial by trial basis when their choice was likely to be erroneous, and used to opt-out in those trials when this was an option. In line with this account, the proportion of opt-out responses strongly increased for larger difficulty (Figure $3 \mathrm{~A}$, middle). Overall, this suggests that the selection of the opt-out option reflects the level of confidence at a particular trial. We also confirmed that reaction times provide another indicator of confidence. As expected, in non-optout trials, the median reaction time as a function of stimulus difficulty displayed the classical X-pattern (Kepecs et al., 2008; Urai et al., 2017): reaction time increases for more difficult stimuli in correct trials, and decreased for more difficult stimuli in error trials (Figure $3 \mathrm{~A}$, bottom).

Since both opt-out selection (for opt-out trials) and reaction time (for non-opt-out trials) are robust indicators of choice confidence on a particular trial, then the overall proportion of opt-out responses and median reaction time in a session likely reflect the level of subject self-confidence at the particular period of time. We found that these indicators were largely stable across the 20 sessions at the population level, whereas the overall accuracy displayed a modest improvement likely due to learning effects in the first two days of the experiment (Figure 3B, top panel). However, at an individual level, these measures displayed important fluctuations which provide a window into subject-specific variations in self-confidence. In particular, the autocorrelograms indicated a slow component to the fluctuations in opt-out tendency (Figure 3C). Significant positive correlations between the proportions of opt-out responses in two sessions were seen when these two sessions were separated as much as two-and-a-half days (both for deterministic and stochastic opt-outs; Figure $3 \mathrm{C}$ middle panel). The same tendency was found for the median reaction time series, although the significance of auto-correlation weights did not survive correction for multiple comparison (bottom panel). The autocorrelations in accuracy across sessions was somehow shorter-lived, with significant correlation between two consecutive sessions (top panel). Finally, we investigated the possible coupling between the values of the different psychometric variables in a single session. We found that the tendency to opt-out in deterministic and stochastic stages were very strongly correlated across sessions (average $r$ across subjects: 0.5 ; t-test: $p<10^{-11}$; see Figure $3 \mathrm{D}, \mathrm{F}$ ). No significant mean correlation across sessions between median reaction times and opt-out tendency (either in stochastic or 
deterministic stage; both $p>0.2$ ) were observed. However, the correlation may be weakened because the different difficulty levels inflate the within-session variance of each variable. We thus computed the difficulty-adjusted across-session correlation between reaction time and opt-out selection, which removes the variance linked to difficulty level from each variable (see Methods). This more sensitive analysis revealed a significant across-session correlation between the median reaction time in correct non-optout trials and both the deterministic (mean residual weight $=0.001, p=0.005$; see Figure $3 \mathrm{E}$ ) and stochastic (mean residual weight $=0.001, p=0.0004$ ) opt-out response proportion. The average accuracy in non-optout trials in a session appeared to be uncorrelated with all three proxies for confidence (opt-out tendency in DO, SO; and average RT). Overall, these results suggest that the tendency to opt-out and average reaction time in the perceptual task provide robust markers of a slowly fluctuating subject confidence. Note that a very similar set of results was found for the orientation task (Supplementary Figure 2).
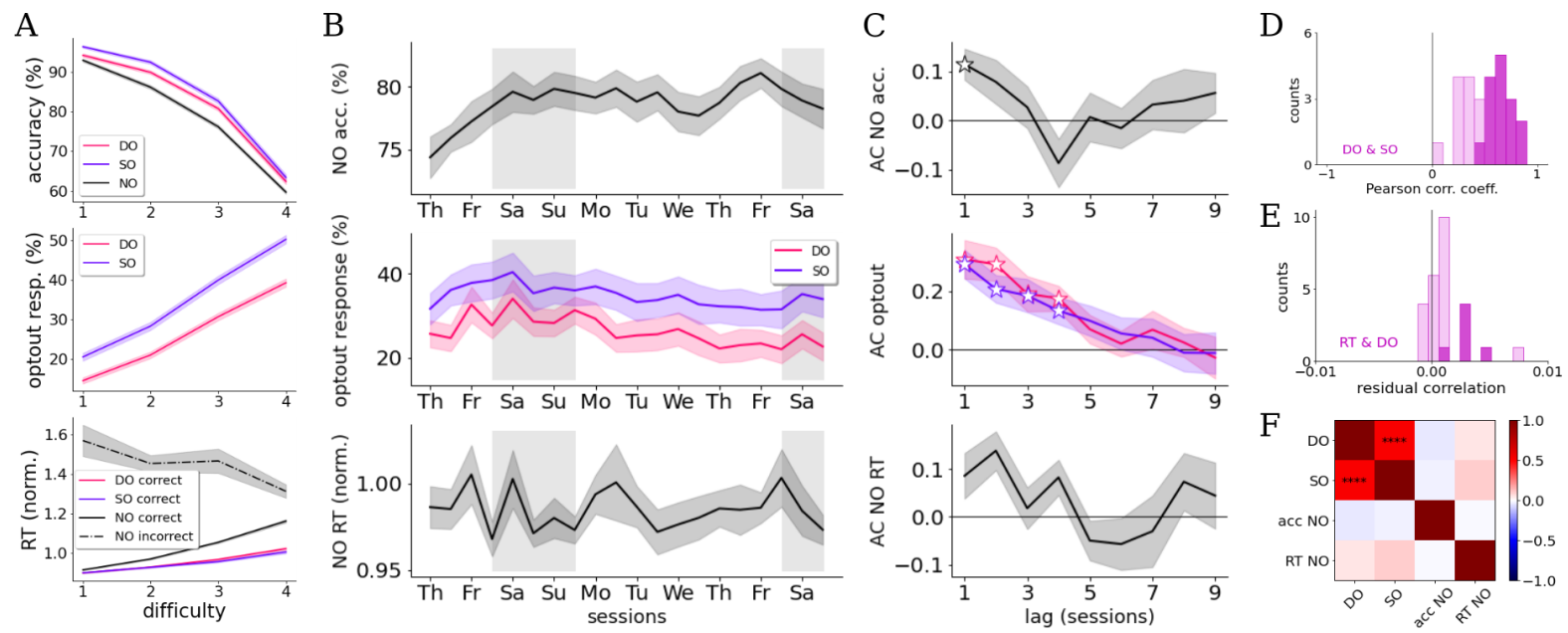

Figure 3. Psychometric variables fluctuations (Numerosity task): A. Mean across participants and sessions, as a function of stimulus difficulty. Top panel: accuracy (percentage in correct responses) in NO (non-optout), DO (deterministic opt-out) and SO (stochastic opt-out) trials. In opt-out trials, the accuracy is expressed as the percentage of correct responses out of the non-optout responses. Middle panel: percentage of opt-out responses, in deterministic and stochastic opt-out trials. Bottom panel: normalized reaction time over correct trials, and incorrect non-optout trials. Reaction times are normalized by the median reaction time across all trials in each session. In all panels shaded areas indicate the standard error of the mean (s.e.m.). B. Psychometric values as a function of session, averaged across participants (shaded area: s.e.m). Gray shaded area indicating weekend sessions. C. Autocorrelation coefficients of accuracy in non-optout trials (top panel), proportion of opt-out responses (middle) and reaction time of correct non-optout trials (bottom). Star: t-test across participants, $p<0.05$ after correction, see Methods. D. Distribution across participants of Pearson coefficients of across-session correlations of the proportion of opt-out responses in deterministic (DO) and stochastic (SO) opt-out stages. Filled bars indicate subjects with significant correlation $(p<0.05)$ at individual level. E. Difficulty-adjusted across-session correlation between reaction time in correct non-optout trials and the proportion of the deterministic opt-out response (see Methods; $n=n_{\text {sessions }} \mathrm{x}$ $\left.n_{\text {difficulties }}=20 \times 4\right)$. Filled bars indicate subjects with significant correlation $(p<0.05)$ at 
individual level. F. Matrix representing the mean correlation coefficient across participants $\left({ }^{* * *} p<0.0001\right.$; significance remains after Bonferroni correction).

\section{A normative approach to over-confidence}

While the proportion of opt-out responses in a session provides an intuitive measure of the confidence level of the participant, we also used a model-based approach based on signal detection theory (SDT) to define a normative measure of overconfidence. According to the SDT framework, in non-opt-out trials there is a single boundary in the observer perceptual space that is used as a decision criterion to select between a leftward or a rightward response. A simple fit to participant responses in non-optout trials allows to infer the decision boundary $H$ as well as the perceptual noise (Figure 4A). When the opt-out option is present, there are now two decision boundaries $H_{L}$ and $H_{R}$ that separates lefwards and opt-out responses for the first, opt-out and rightwards responses for the second. An optimal observer would place these boundaries such as to maximize the expected number of points. For example, in the deterministic opt-out stage where correct responses are rewarded by 3 points while opt-outs yield 2 points, the optimal boundary $H_{L}$ is placed at the point in the perceptual space corresponding to a $2 / 3$ probability of a leftward stimulus (and similarly for $H_{R}$ ). The position of this point can be evaluated analytically and depends on the value of the perceptual noise (see Methods for details). We can then estimate boundaries $H_{L}$ and $H_{R}$ from responses in the opt-out condition and compare the distance between these estimated boundaries to the distance between the optimal boundaries (Figure 4B). A larger than optimal inter-boundary distance reflects a larger than optimal use of the opt-out option, i.e. can be associated with an underestimation in the probability of a correct judgment. By contrast, a smaller than optimal inter-boundary distance reflects less frequent than optimal resorting to the opt-out response, which signals an overestimation of the probability for a correct judgment. We define an overconfidence metrics (see Methods) based on the relationship of the estimated inter-boundary distance to the optimal inter-boundary distance, as a metric bounded between -1 and 1 , where 0 reflects optimal distance, positive values reflect overconfidence and negative values reflect underconfidence (see fluctuations on overconfidence across sessions in Supplementary Figure 3).

We estimated decision boundaries and perceptual noise from the pattern of responses in non-optout, deterministic opt-out and stochastic trials in each session and each participant (Figure 4C,F). The SDT framework predicts that only the position of boundaries should vary between opt-out and non-optout trials, while the perceptual noise should not. Indeed we found a strong correlation between the perceptual noise estimated from opt-out and non-optout trials belonging to the same session (Figure 4E). This was observed for deterministic as well as stochastic opt-out trials, and for both NT and OT. We also predict that the decision bias should be preserved in opt-out trials. If the value of the decision boundary $H$ reflects a bias of the mapping of the stimulus onto the perceptual space (e.g. if the number of dots in the left circle are consistently underestimated), then boundaries $H_{L}$ and $H_{R}$ should be similarly biased. Indeed we also found a strong correlation across sessions between the bias in the non-optout trials -defined simply as $\mathrm{H}$ - and the bias in the opt-out trials -defined as the middle point between $H_{L}$ and $H_{R}$ - (Figure 4D). These analyses confirm that SDT provides a sound framework for understanding participant responses, and validate our normative approach to overconfidence derived from this framework. 
A
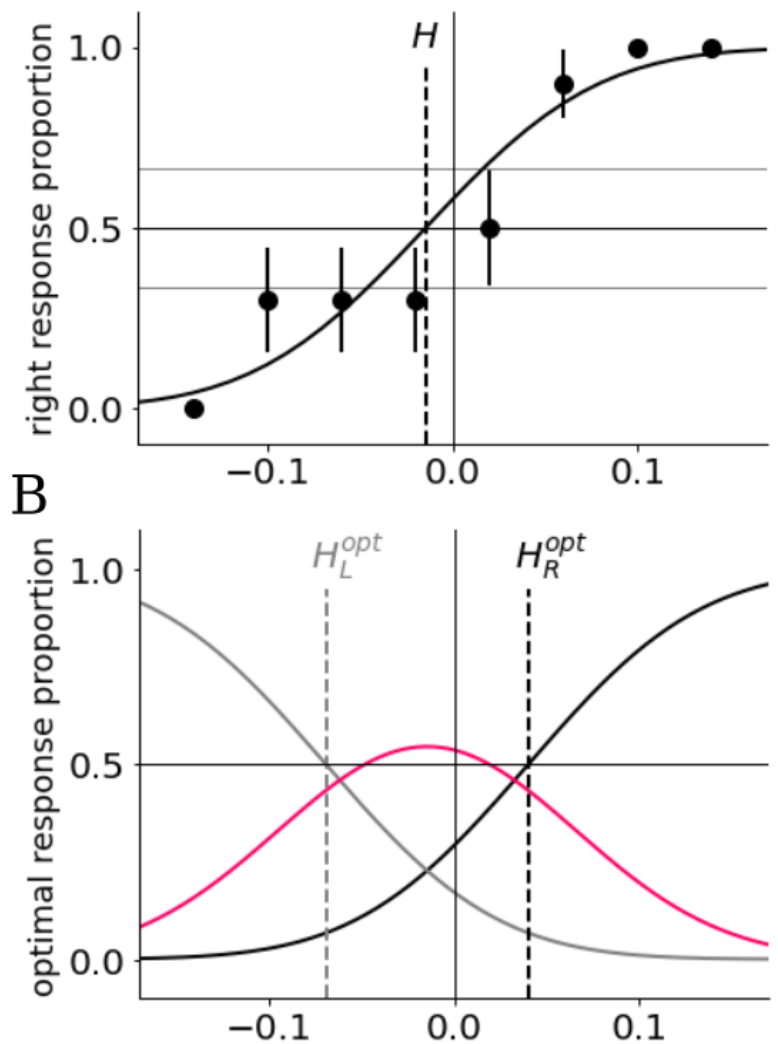

C

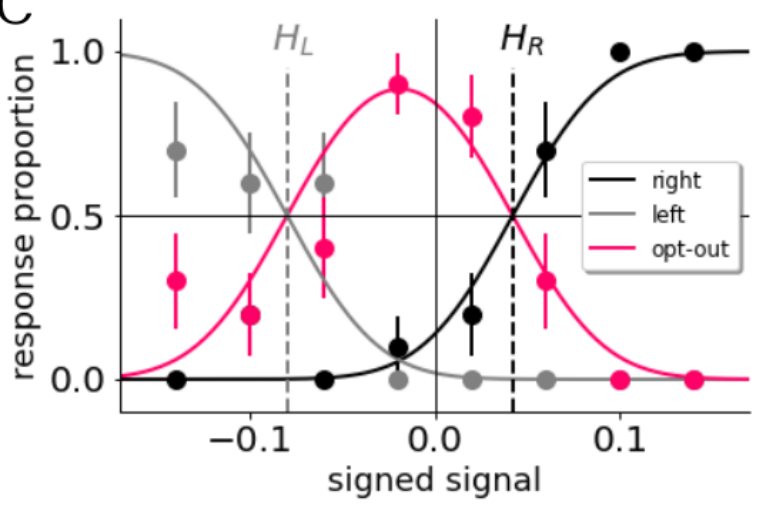

$\mathrm{D}$

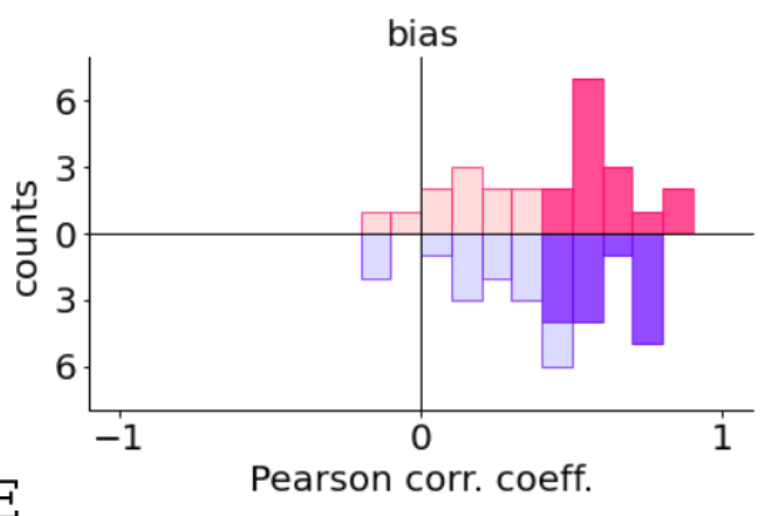

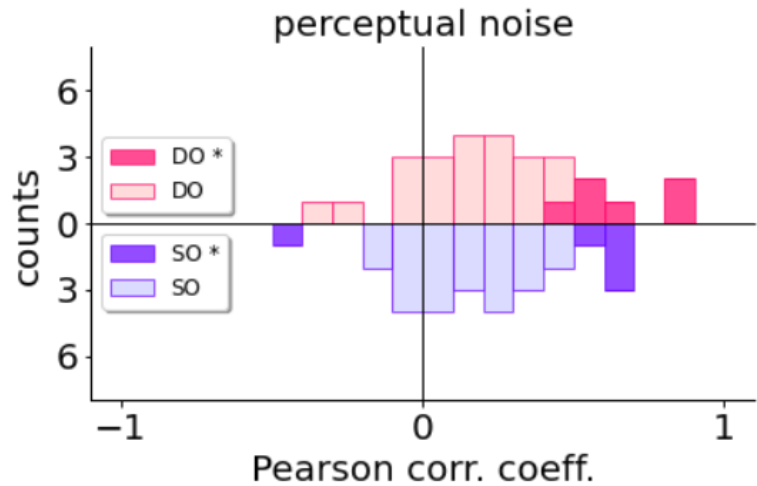

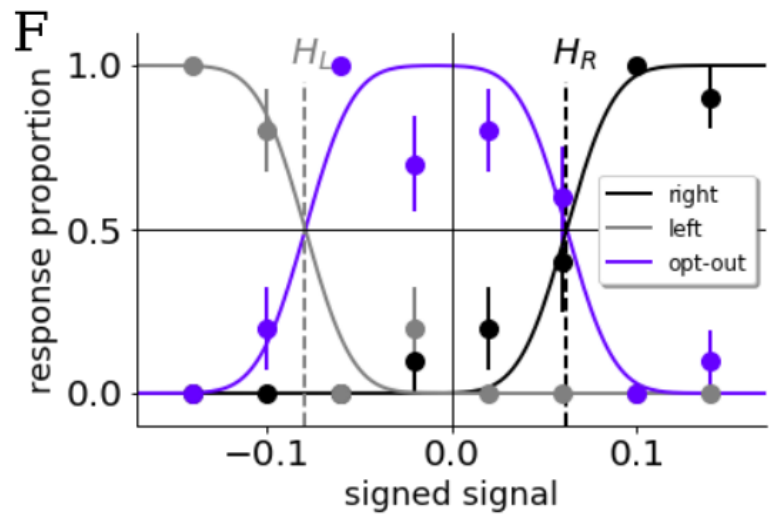

Figure 4. Psychometric curves. A. Example psychometric curve in non-optout trials in one session for a participant performing the numerosity task. The sigmoid reflects the fit by a probit sigmoid function ( $H$ : estimated decision boundary; see methods). B. Optimal psychometric curves inferred for deterministic opt-out trials of this participant-session. The grey, red and black curves represent the probability of leftwards, opt-out and rightwards responses, respectively ( $H_{L}^{\text {opt }}$ and $H_{R}^{\text {opt }}$ : left and right optimal decision boundaries). C, F. Example of the psychometric curve in deterministic $(C)$ and stochastic $(F)$ opt-out trials for the same participant and session as in panel A. Lines representing the psychometric fits for each type of choice $\left(H_{L}\right.$ and $H_{R}$ : left and right decision boundaries). D, E. Distribution of across-session Pearson correlation coefficient of bias (D) and perceptual noise (E) between non-optout and opt-out trials (DO: red/upright histogram; SO: blue/inverted histogram) in the numerosity task. Filled bars indicate subjects with significant correlation $(p<0.05)$ at individual level. 


\section{Emotional states and self-confidence fluctuate independently}

In the prior analyses, we have characterized the dynamics and relationships between emotional states on one hand, and between psychometric markers on the other hand. We finally evaluated whether the evolution of self-reports and psychometric markers were coupled. In particular, we hypothesized that mood correlates across sessions with the different proxies for self-confidence. At odds with our hypothesis, at the population level, we found no correlation across sessions between the self-confidence proxies (proportion of opt-out choice, median reaction times) derived from the perceptual tasks and mood or any of the other quality of life self-reports. The correlation between mood and median reaction time was negatively correlated, as predicted (mean $r=-0.09$ ), but the marginal significance (ttest, $p=0.03$ uncorrected) did not survive Bonferroni correction for multiple comparison (Figure $5 \mathrm{~A}$ ). To further test if the correlation could emerge with a specific dimension of the opt-out behavior, we separated the stimulus-insensitive and stimulus-sensitive portions of the opt-out responses in each session by extracting the slope and the intercept of a simple linear regression of the opt-out proportion by stimulus difficulty. Neither the intercepts nor the slopes correlated across sessions with any of the quality of life reports. Furthermore, we reasoned that our normative definition of overconfidence may provide a better proxy to self-confidence than the raw proportion of opt-out responses in the session, and so we also tested for correlations of the overconfidence index with the quality of life self-reports. None of the correlations were significant. Self-reports were also not coupled significantly with other psychometric variables such as the overall accuracy (for non-optout trials) in the session, or the degree of risk aversion. Overall, we did not find any significant mean correlation between a self-report and a psychometric variable.

At the individual level, however, we found that a significant subset of participants (4 out of 27 in NT, binomial test with $p=0.01$ uncorrected) exhibited significant correlation between the proportion of opt-out choice and mood: two subjects opted out significantly less in sessions where they reported better mood, while two other subjects opted out significantly more (Figure 5B top-left panel). This result could indicate a subject-specific relationship between mood state and the level of confidence. This pattern was not found in the OT (no significant correlation in any participant). We aimed at confirming this possible subject-dependent relationship between confidence and mood by using a more principled approach. We defined a Bayesian mixed model (or Bayesian hierarchical model) where the confidence proxy in the session was used as the dependent variable and participant was taken as the random factor. In other words this model included both subject-specific and subject-unspecific sensitivity of the confidence proxy to the mood report. This model was compared to a control Bayesian mixed model that included neither of these two terms. Bayesian modelling allows us to potentially obtain evidence not only against the null hypothesis, but also in favor of it. Model comparison using the WAIC criterion confirmed that there is moderate evidence for a subject-specific influence of mood onto confidence proxies (difference in WAIC for explaining the proportion of opt out responses in the NT: 3.3; for explaining median reaction time: 1.0; see Supp. fig. 4). Similar results were found for the influence of stress over self-confidence (difference in WAIC for optout: 11.2; for explaining median reaction time: 1.0). Using an alternative criterion (LOO-CV) provided very similar results. Moreover, the $r^{2}$ of the different mixed models was not much larger than the $r^{2}$ of the respective control models ( $r^{2}$ difference of 0.01 for the association between either confidence proxy and mood report in NT). In other words, incorporating the subject-specific effect of mood (or other reports) only marginally increased the how well the confidence proxy could be predicted (see Supp. fig. 5). 
Finally, we investigated whether self-confidence and mood states could be coupled but with a certain delay by computing the cross-correlation between the proportion of opt-out responses and the different self-report measures. The cross-correlograms were all flat (Figure $5 \mathrm{C}$ ), indicating an absence of coupling at the population level between emotional states and self-confidence proxies. 
A
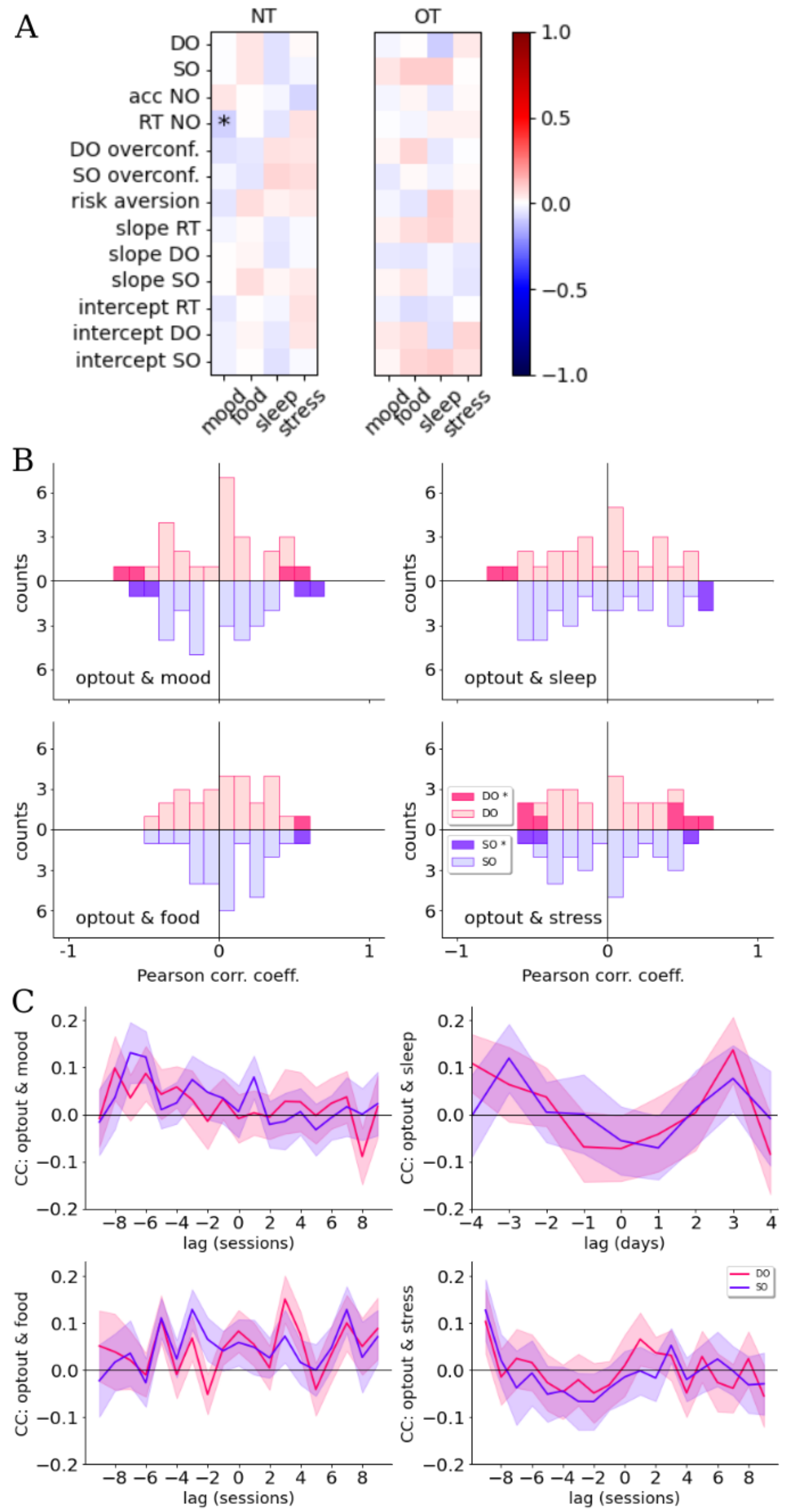
Figure 5. Lack of robust correlation between self-reports \& psychometric variables $\mathbf{A}$. Mean of correlation coefficients between self-report and psychometric variables (proportion of opt-out responses in DO stage, proportion of opt-out responses in SO stage, mean accuracy in non-optout trials, median reaction time in correct non-optout responses, overconfidence in DO stage, overconfidence in SO stage, risk aversion, stimulus-insensitive and stimulus-sensitive components of opt-out responses), averaged across participants ( ${ }^{*} p$ $<0.05$ uncorrected) for both the numerosity and (NT) the orientation (OT) task groups. B. Distribution of Pearson coefficients of correlation between the proportion of opt-out responses (deterministic: red/upright histogram; stochastic: blue/inverted histogram) and each self-report along sessions for the NT group. Filled bars indicate subjects with significant correlation $(p<0.05)$ at individual level. C. Cross-correlation coefficients between both opt-out election types (DO in solid line and SO in dashed line) and each self-report for the NT group.

\section{Discussion}

In this longitudinal online study, we assessed for the first time whether daily fluctuations in mood and related variables (stress, sleep, food enjoyment) are coupled to fluctuations in metacognitive states (including self-confidence, response vigor, discrimination performance and risk aversion). Participants directly reported their emotional states at the beginning of each bi-daily sessions, while metacognitive states were inferred from the behavior in a simple discrimination task with an opt-out option (Figure 1). First, we found a strong correlation between the different mood-related variables, visible at the level of individual participants (Figure 2). The different reports fluctuated rapidly, as the value of one session correlated significantly with the value half-a-day later but not one day later (except for food enjoyment). Second, the proportion of opt-out responses and the median reaction time on non-optout trials provided solid markers of the confidence of the participant in a particular session (Figure 3). We also derived participant overconfidence in a normative setting based on the signal detection theory framework (Figure 4). Self-confidence fluctuated at a slow time scales, with auto-correlations of the time series with a lag up to two-and-a-half days. Finally, none of the mood-related variables correlated significantly with any metacognitive variable in the same session at the population level (Figure 5). In particular, mood did not appear to correlate with self-confidence. An exploratory analysis revealed however that the correlation between mood and the proportion of opt-out responses was found at the individual level in a significant fraction of participants in the NT. Overall, our results suggest that mood-related states and metacognitive states fluctuate independently in the healthy adult, with slower fluctuations for self-confidence than for the mood-related variables.

Our results related to the discrimination task follow a long tradition of collecting implicit markers of metacognitive states (including confidence) from behavioral measures. Confidence that humans have in decision-making tasks is often measured by asking them to explicitly report their confidence in a decision or series of decisions (Martino et al., 2013; Schustek et al., 2019). However, these reports have some drawbacks: they are not intuitive, participants may feel little motivation to report accurately their confidence level, and they can be contaminated by learning processes (Solovey et al., 2016). Importantly, emotional states and mood in particular may interfere with the process of explicitly reporting a confidence measure. Here, we used an implicit measure of confidence in order to test core relationships 
between confidence and mood states without potential contamination by the reporting process. Implicit markers of confidence rely on an economic paradigm where confidence is linked to the willingness to bet on their own choice and the magnitude of the bet (Grimaldi et al., 2015; Insabato et al., 2016; Sanders et al., 2016). We used an opt-out mechanism where in a portion of trials participants could, instead of reporting the stimulus category, secure a fixed number of points (DO stage) or bet on a lottery (SO stage). Such opt-out or wagering scheme has been used in a large range of settings, from perceptual discrimination in non-human primates (Kiani \& Shadlen, 2009), rodents (Kepecs et al., 2008) and humans (García-Pérez \& Alcalá-Quintana, 2017) to movie selection in adults (Bhatia \& Mullett, 2016). In line with these studies, we found that the use of opt-out responses in our participants could be tied to the expected accuracy in the discrimination, i.e. the confidence about the choice. First, resorting to the opt-out increased with stimulus difficulty, when expected accuracy is bound to decrease. Second, for a fixed stimulus difficulty, the accuracy of responses increased when the opt-out response was presented but waived compared to trials where the opt-out response was not presented. This suggests that participants selected the opt-out response when their internal estimate of the stimulus category did not ensure a high probability of correct response, thus avoiding potential errors (Kiani \& Shadlen, 2009). We also used reaction time in non-optout trials as another proxy for self-confidence (Martino et al., 2013; Moreno-Bote, 2010; Vickers, 1979). We replicated the findings that reaction times in such discrimination task display the typical X-pattern expected of a confidence proxy, with faster responses associated with larger stimulus strenght in correct trials, but slower responses associated with larger sitmulus strength in error trials (Sanders et al., 2016; Urai et al., 2017). Spontaneous daily variations in confidence have to our knowledge never been reported (see (Morphew, 2021) for slower variations). Here, both proxies of confidence fluctuated slowly across sessions, with a time scale of up to two days (4 sessions). Of note, the two confidence proxies (proportion of opt-out responses and median reaction time) were weakly but significantly correlated across sessions (Figure 3E). This suggests that they might at least partially report on the confidence construct. It is also possible that the weak correlation was due to reaction times being also influenced by other factors such as vigor or the particular position of the hand of the subject on the keyboard.

We introduced a normative estimation of overconfidence that assesses how much participants resort to the opt-out option compared to what would be optimal given their level of perceptual noise (Moore \& Healy, 2008). The definition is based on the framework of signal detection theory (Barrett et al., 2013; Massoni et al., 2014). We confirmed beforehand that signal detection theory provided a reasonable account of the pattern of choices both in non-optout and opt-out trials. In opt-out trials, we assumed that the decision space is splitted according to two boundaries: the boundary between left and opt-out responses, and the boundary between opt-out and right responses (García-Pérez \& Alcalá-Quintana, 2010, 2017; Pritchett \& Murray, 2015). We also found that both the level of perceptual noise and the bias were consistent between the opt-out and non-optout trials in the same session. The overconfidence is based on comparing the distance between decision boundaries used by participants and the distance between optimal boundaries, which depends on the level of perceptual noise. Thus, underestimating the perceptual noise leads to overconfidence, as measured by our normative index. We believe this definition offers a normative approach to measuring implicitly the level of overconfidence in healthy subjects and pathological populations. 
Here, we tested for the first time, to our knowledge, the possible link between spontaneous fluctuations in mood states and confidence in the healthy adult population. No significant correlation at the group level was found between the reported quality of life states and the implicit self-confidence markers extracted from the decision-making task. It is important to note that these non-significant effects are probably not related to a lack of statistical power in our data, as some temporal correlations between quality of life states and between self-confidence markers reached very strong significance levels. The autocorrelograms of the different variables revealed that confidence proxies fluctuated at a slower time scale (up to two-and-a-half days) than mood states (half-a-day). Altogether, we found support for fluctuations of mood and confidence with different dynamical properties and little to no interaction. We did find however a small subset of participants that displayed significant correlations (some positive, some negative) between mood reports and the proportion of opt-out responses in the NT cohort. This suggests that a subject-specific link between mood and confidence may exist in some individuals. Indeed, a bayesian mixed model incorporating such subject-specific influence of mood report on confidence proxy better accounted for the experimental data than a control model without such influence. The difference was however modest, both in terms of model selection metrics and variance explained. Testing on a larger cohort would be needed to confirm this exploratory finding and explore the demographic or personality features associated with such a link.

The absence of a consistent link suggested by this study defies our original hypothesis which was based on a series of adjacent findings. For example, Massoni showed that anxiety about the future performance in the numerosity task affects metacognition on that task, and was in particular associated with a lower level of overconfidence (Massoni, 2014). Three key differences in the paradigm could explain the discrepancies of the results: i) in his experiment, anxiety was expressed towards the task specifically, while participants in our experiment report their mood irrespective of the task (and prior to performing it); ii) anxiety was induced by the paradigm in his study, while we only measured spontaneous variations in mood: iii) anxiety in his study was a short-lived emotional state, evolving from one block of trials to the other, while reported mood states in our experiment fluctuated over days. It has also long been known that induced sadness reduces risk aversion, while induced anxiety enhances it (Hartley \& Phelps, 2012; Raghunathan \& Pham, 1999); and that momentary mood modulates the valuation of both positive and negative outcomes when subjects gamble or decide to opt in or out a challenging task (Eldar \& Niv, 2015; Vinckier et al., 2018). Future studies are needed to understand which of these factors are key to the association between emotional states and metacognitive states including confidence: the spontaneous vs. induced nature of the emotional state, the time scale of its fluctuations (minutes, days or years), and the attachment of the emotion to the task. Note that while we looked for within-subject coupling between emotional states and metacognition, there are clear signs of across-subject associations between psychopathological symptoms and metacognitive abilities. For example, a symptom dimension related to anxiety and depression correlates with lower confidence and heightened metacognitive efficiency on the numerosity task (Rouault et al., 2018), while stable anxiety traits are also associated with increased risk aversion (Maner et al., 2007). We also failed to find any link between natural daily fluctuations in either mood or stress level and risk aversion (Figure 5). This contrasts with previous findings which found that unexpected positive events (such as good weather or a sports win) increase risk-seeking behavior (Bassi et al., 2013.; Edmans et al., 2007.; Otto et al., 2016) and that the effect was mediated through improving mood (Bassi et al., 2013) . 
The lack of a consistent correlation between daily fluctuations of mood and metacognition comes also as a surprise given the strong association between deficits in emotional and metacognitive processes in several psychiatric disorders. For example, depressed patients, which primarily suffer from sustained negative mood states, also display increased underconfidence in their own decisions (Fu et al., 2005). On the contrary, schizophrenic patients, who suffer from dysregulated mood, generally express strong overconfidence in their own decisions and poor insight about their dysfunctions (David et al., 2014), a phenomenon usually linked to circular inference (Jardri et al., 2017; Jardri \& Denève, 2013). Again, we can only speculate about why these associations are present in psychiatric disorders but apparently absent in spontaneous fluctuations in the healthy population. The link may be only present in pathological conditions, or for very slow and stable features (i.e. the association may be across individuals but not within individuals). Given the very diverse etiology of these different disorders, it is also possible that the mood and metacognitive dysregulations represent independent manifestations of the disorder.

Beyond confidence and risk aversion, other markers of cognitive function are thought to be affected by mood states, quality of sleep and the diurnal rhythm (Leone et al., 2017). For example, extreme sleep deprivation and stress exposure leads to a decrease in perceptual task performance and slowing of the responses (Lieberman et al., 2002). We found no sign of such relation between spontaneous and moderate fluctuations of sleep and stress and either the accuracy or speed of responses. More recently, lower quality of sleep in the general population was found to abolish the differential sensitivity to risk aversion under gain and loss frames (S. Xu et al., 2021). This behavioral result was mirrored by a reduction in the bad sleepers of the difference between the EEG responses to negative feedback in the gain vs loss frames. Moreover, depression is associated both with lower mood and reduced motor vigor (Carland et al., 2019; Van De Leemput et al., 2014). In constrast to these results, we did not find any relationship between spontaneous fluctuations in mood and response times in a discrimination task.

Our experimental design aimed at tracking longitudinally the evolution of affective and metacognitive states of subjects in ecologically valid conditions, in order to unveil how these dimensions evolve and co-evolve over a period of two weeks. Findings in this area could contribute to a better understanding of affective states disorders. Indeed, the standard clinical symptom-based categorization of affective disorders has been widely criticised due to the absence of objective diagnostic criteria that take into account the neurobiology of affective states and the broader context in which they take place during the life of an individual (Hyman, 2010). Novel approaches to the diagnosis of mental disorders, such as the RDoC (Insel, 2014) support an holistic view on mental health that stresses the importance of analyzing affective states longitudinally at multiple scales and multiple time frames. Empirical studies(Van De Leemput et al., 2014) found that in the transition to depressed state, the mood of patients fluctuates more slowly (e.g. higher autocorrelation) and correlates negatively with anxiety, a result compatible with the negative relationship between mood and stress reported in our data. Similarly, recent studies characterized depression as the result of an interaction between micro- and macro-behavioural patterns repeated over time (Wichers, 2014), a result also confirmed by computational modelling of longitudinal self-reports (Bringmann et al., 2013). Our findings are consistent with these results as we find that mood fluctuations are predictable over time and strongly correlate with 
fluctuations of other mental and physical dimensions (e.g. appetite and sleep). In that we extended the notion of wellbeing from the purely affective domain to a broader spectrum that includes both mental and physical states and points at an underlying holistic well-being model comprising multiple dimensions and timescales (Rutledge et al., 2014).

In the last decades, the growing smartphone adoption has enabled the collection of longitudinal datasets of affective states in the wild (Gillan \& Rutledge, 2021; Sano et al., 2015; Taquet et al., 2020, 2021; Taylor et al., 2017). Indeed, daily fluctuations of emotional states are very well reported through smartphones during longitudinal studies involving large cohorts over longer periods of time (Moturu et al., 2011; Sano et al., 2015; Triantafillou et al., 2019). These studies support the validity of mobile technology to track mental wellbeing and show for example a predictive relationship between sleep quality and mood, with poor sleepers reporting high PSS (self-reported Perceived Scale Stress) and subjective low happiness (Wild-Hartmann et al., 2013). Our dataset, collected with a similar online platform in the wild, supports these findings showing a significant relationship between sleep quality, stress level and mood (Moturu et al., 2011; Sano et al., 2015; Triantafillou et al., 2019). We complement these findings by showing that the enjoyment of food is linked with mood, stress levels and quality of sleep.

A relevant application that motivates the longitudinal study of affective and cognitive states in the wild is the development of digital phenotyping models to infer physical and mental conditions and drive personalized treatment (Huckvale et al., 2019; Insel, 2018). Digital phenotyping aims at tracking and modelling behavioral variables of subjects via passive smartphone sensing with the purpose of extracting digital biomarkers predictive of physical and mental health (Torous \& Firth, 2016). Importantly, the majority of digital phenotyping applications rely on passive smartphone sensing to extract correlates of affective and mental states based on behavioral markers, but do not attempt at accessing explicitly any cognitive dimension (Marsch, 2020). Advances in the study of decision making and metacognition will potentially foster more sophisticated digital phenotyping applications by enhancing passive data collection with the active delivery of decision making tasks. Indeed, the principles on which our decision making task is based can be embedded into games or recommender systems interfaces to implicitly test the way subjects take perceptual or value based decisions and use the extracted decision making parameters to infer affective and mental states (Brown et al., 2014).

\section{Acknowledgements}

We thank Aleksandar Matic for his contribution to designing the project and for very creative discussions about the results; Arnau Masdevall-Lara and Silvia Pérez-García for their help on developing the experimental paradigms, collecting and analysing pilot data; Guillermo Solovey and Andrés Taraciuk for productive discussions on the experimental code development. MdF was funded by grant "Linking Mood and Metacognition through a mobile based experimental platform" from Koa Heath B.V. (formerly Telefonica Innovation Alpha). RM-B is supported by BFU2017-85936-P from MINECO (Spain), the Howard Hughes Medical Institute (HHMI; ref 55008742), an ICREA Academia award, and the Bial Foundation (grant number 117/18). AH is funded by the Spanish Ministry of Economy (grants PSI-2015-74644-JIN from Jovenes-Investigadores programme and RYC-2017-23231 from Ramon y Cajal programme). 


\section{Conflict of interest}

The researchers collaborated in the development of the Koa Health B.V. digital products. GM received salary support from Koa Health B.V.

\section{References}

Averbeck, B. B., Evans, S., Chouhan, V., Bristow, E., \& Shergill, S. S. (2011). Probabilistic learning and inference in schizophrenia. Schizophrenia Research, 127(1-3), 115-122.

Barrett, A. B., Dienes, Z., \& Seth, A. K. (2013). Measures of metacognition on signal-detection theoretic models. In Psychological Methods (Vol. 18, Issue 4, pp. 535-552). https://doi.org/10.1037/a0033268

Bassi, A., Colacito, R., \& Fulghieri, P. (2013). 'O Sole Mio: An Experimental Analysis of Weather and Risk Attitudes in Financial Decisions. In SSRN Electronic Journal. https://doi.org/10.2139/ssrn.1958215

Bhatia, S., \& Mullett, T. L. (2016). The dynamics of deferred decision. Cognitive Psychology, 86, 112-151.

Bringmann, L. F., Vissers, N., Wichers, M., Geschwind, N., Kuppens, P., Peeters, F., Borsboom, D., \& Tuerlinckx, F. (2013). A Network Approach to Psychopathology: New Insights into Clinical Longitudinal Data. In PLoS ONE (Vol. 8, Issue 4, p. e60188). https://doi.org/10.1371/journal.pone.0060188

Brown, H. R., Zeidman, P., Smittenaar, P., Adams, R. A., McNab, F., Rutledge, R. B., \& Dolan, R. J. (2014). Crowdsourcing for Cognitive Science - The Utility of Smartphones. PloS One, 9(7), e100662.

Carland, M. A., Thura, D., \& Cisek, P. (2019). The Urge to Decide and Act: Implications for Brain Function and Dysfunction. The Neuroscientist: A Review Journal Bringing Neurobiology, Neurology and Psychiatry, 25(5), 491-511.

Dagum, P. (2018). Digital biomarkers of cognitive function. Npj Digital Medicine, 1(1), 10.

Damasio, A. (2008). Descartes'Error: Emotion, Reason and the Human Brain. Random House.

David, A. S., Bedford, N., Wiffen, B., \& Gilleen, J. (2014). Failures of Metacognition and Lack of Insight in Neuropsychiatric Disorders. In The Cognitive Neuroscience of Metacognition (pp. 345-365). https://doi.org/10.1007/978-3-642-45190-4_15

Dienes, Z., \& Seth, A. (2010). Gambling on the unconscious: A comparison of wagering and confidence ratings as measures of awareness in an artificial grammar task. In Consciousness and Cognition (Vol. 19, Issue 2, pp. 674-681). https://doi.org/10.1016/j.concog.2009.09.009

Edmans, A., Garcia, D., \& Norli, O. (2007). Sports Sentiment and Stock Returns. In SSRN Electronic Journal. https://doi.org/10.2139/ssrn.677103

Eldar, E., \& Niv, Y. (2015). Interaction between emotional state and learning underlies mood instability. Nature Communications, 6, 6149 . 
Eldar, E., Roth, C., Dayan, P., \& Dolan, R. J. (2018). Decodability of Reward Learning Signals Predicts Mood Fluctuations. Current Biology: CB, 28(9), 1433-1439.e7.

Eldar, E., Rutledge, R. B., Dolan, R. J., \& Niv, Y. (2016). Mood as Representation of Momentum. Trends in Cognitive Sciences, 20(1), 15-24.

Fleming, S. M., Massoni, S., Gajdos, T., \& Vergnaud, J.-C. (2016). Metacognition about the past and future: quantifying common and distinct influences on prospective and retrospective judgments of self-performance. Neuroscience of Consciousness, 2016(1). https://doi.org/10.1093/nc/niw018

Fu, T., Koutstaal, W., Fu, C. H. Y., Poon, L., \& Cleare, A. J. (2005). Depression, Confidence, and Decision: Evidence Against Depressive Realism. In Journal of Psychopathology and Behavioral Assessment (Vol. 27, Issue 4, pp. 243-252). https://doi.org/10.1007/s10862-005-2404-x

García-Pérez, M. A., \& Alcalá-Quintana, R. (2010). THE DIFFERENCE MODEL WITH GUESSING EXPLAINS INTERVAL BIAS IN TWO-ALTERNATIVE FORCED-CHOICE DETECTION PROCEDURES. In Journal of Sensory Studies (Vol. 25, Issue 6, pp. 876-898). https://doi.org/10.1111/j.1745-459x.2010.00310.x

García-Pérez, M. A., \& Alcalá-Quintana, R. (2017). The Indecision Model of Psychophysical Performance in Dual-Presentation Tasks: Parameter Estimation and Comparative Analysis of Response Formats. In Frontiers in Psychology (Vol. 8). https://doi.org/10.3389/fpsyg.2017.01142

Gillan, C. M., \& Rutledge, R. B. (2021). Smartphones and the Neuroscience of Mental Health. Annual Review of Neuroscience. https://doi.org/10.1146/annurev-neuro-101220-014053

Grimaldi, P., Lau, H., \& Basso, M. A. (2015). There are things that we know that we know, and there are things that we do not know we do not know: Confidence in decision-making. Neuroscience and Biobehavioral Reviews, 55. https://doi.org/10.1016/j.neubiorev.2015.04.006

Hartley, C. A., \& Phelps, E. A. (2012). Anxiety and Decision-Making. In Biological Psychiatry (Vol. 72, Issue 2, pp. 113-118). https://doi.org/10.1016/j.biopsych.2011.12.027

Hoven, M., Lebreton, M., Engelmann, J. B., Denys, D., Luigjes, J., \& van Holst, R. J. (2019). Abnormalities of confidence in psychiatry: an overview and future perspectives. Translational Psychiatry, 9(1), 268.

Huckvale, K., Venkatesh, S., \& Christensen, H. (2019). Toward clinical digital phenotyping: a timely opportunity to consider purpose, quality, and safety. NPJ Digital Medicine, 2, 88.

Hyman, S. E. (2010). The Diagnosis of Mental Disorders: The Problem of Reification. In Annual Review of Clinical Psychology (Vol. 6, Issue 1, pp. 155-179). https://doi.org/10.1146/annurev.clinpsy.3.022806.091532 Insabato, A., Pannunzi, M., \& Deco, G. (2016). Neural correlates of metacognition: A critical perspective on current tasks. In Neuroscience \& Biobehavioral Reviews (Vol. 71, pp. 167-175). https://doi.org/10.1016/j.neubiorev.2016.08.030

Insel, T. R. (2014). The NIMH Research Domain Criteria (RDoC) Project: precision medicine for psychiatry. The American Journal of Psychiatry, 171(4), 395-397. 
Insel, T. R. (2018). Digital phenotyping: a global tool for psychiatry. World Psychiatry: Official Journal of the World Psychiatric Association , 17(3), 276-277.

Jardri, R., \& Denève, S. (2013). Circular inferences in schizophrenia. Brain: A Journal of Neurology. https://doi.org/10.1093/brain/awt257

Jardri, R., Duverne, S., Litvinova, A. S., \& Denève, S. (2017). Experimental evidence for circular inference in schizophrenia. Nature Communications, 8(1), 1-13.

Jones, N. M., Johnson, M., Sathappan, A. V., \& Torous, J. (2021). Benefits and Limitations of Implementing Mental Health Apps Among the Working Population. Psychiatric Annals, 51(2), 76-83.

Kepecs, A., Uchida, N., Zariwala, H. A., \& Mainen, Z. F. (2008). Neural correlates, computation and behavioural impact of decision confidence. In Nature (Vol. 455, Issue 7210, pp. 227-231). https://doi.org/10.1038/nature07200

Kiani, R., \& Shadlen, M. N. (2009). Representation of confidence associated with a decision by neurons in the parietal cortex. Science, 324(5928), 759-764.

Lange, K., Kühn, S., \& Filevich, E. (2015). “Just Another Tool for Online Studies” (JATOS): An Easy Solution for Setup and Management of Web Servers Supporting Online Studies. In PLOS ONE (Vol. 10, Issue 6, p. e0130834). https://doi.org/10.1371/journal.pone.0130834

Larsen, R. J., \& Kasimatis, M. (1990). Individual differences in entrainment of mood to the weekly calendar. In Journal of Personality and Social Psychology (Vol. 58, Issue 1, pp. 164-171). https://doi.org/10.1037/0022-3514.58.1.164

Leone, M. J., Fernandez Slezak, D., Golombek, D., \& Sigman, M. (2017). Time to decide: Diurnal variations on the speed and quality of human decisions. Cognition, 158, 44-55.

Lieberman, H. R., Tharion, W. J., Shukitt-Hale, B., Speckman, K. L., \& Tulley, R. (2002). Effects of caffeine, sleep loss, and stress on cognitive performance and mood during U.S. Navy SEAL training. Psychopharmacology, 164(3), 250-261.

Maner, J. K., Anthony Richey, J., Cromer, K., Mallott, M., Lejuez, C. W., Joiner, T. E., \& Schmidt, N. B. (2007). Dispositional anxiety and risk-avoidant decision-making. In Personality and Individual Differences (Vol. 42, Issue 4, pp. 665-675). https://doi.org/10.1016/j.paid.2006.08.016

Marriott, F. H. C., \& Pope, J. A. (1954). Bias in the Estimation of Autocorrelations. In Biometrika (Vol. 41, Issue 3/4, p. 390). https://doi.org/10.2307/2332719

Marsch, L. A. (2020). Digital health data-driven approaches to understand human behavior. Neuropsychopharmacology: Official Publication of the American College of Neuropsychopharmacology, 46(1), 191-196.

Martino, B. D., De Martino, B., Fleming, S. M., Garrett, N., \& Dolan, R. J. (2013). Confidence in value-based choice. In Nature Neuroscience (Vol. 16, Issue 1, pp. 105-110). https://doi.org/10.1038/nn.3279 
Massoni, S. (2014). Emotion as a boost to metacognition: how worry enhances the quality of confidence. Consciousness and Cognition, 29, 189-198.

Massoni, S., Gajdos, T., \& Vergnaud, J.-C. (2014). Confidence measurement in the light of signal detection theory. Frontiers in Psychology, 5, 1455.

Moore, D. A., \& Healy, P. J. (2008). The trouble with overconfidence. Psychological Review, 115(2), 502-517.

Moreno-Bote, R. (2010). Decision confidence and uncertainty in diffusion models with partially correlated neuronal integrators. Neural Computation, 22(7), 1786-1811.

Morphew, J. W. (2021). Changes in metacognitive monitoring accuracy in an introductory physics course. In Metacognition and Learning (Vol. 16, Issue 1, pp. 89-111). https://doi.org/10.1007/s11409-020-09239-3

Moturu, S. T., Khayal, I., Aharony, N., Pan, W., \& Pentland, A. S. (2011). Sleep, mood and sociability in a healthy population. Conference Proceedings: ... Annual International Conference of the IEEE Engineering in Medicine and Biology Society. IEEE Engineering in Medicine and Biology Society. Conference, 2011, $5267-5270$.

Moutoussis, M., Bentall, R. P., El-Deredy, W., \& Dayan, P. (2011). Bayesian modelling of Jumping-to-Conclusions bias in delusional patients. Cognitive Neuropsychiatry, 16(5), 422-447.

Otto, A. R., Fleming, S. M., \& Glimcher, P. W. (2016). Unexpected but Incidental Positive Outcomes Predict Real-World Gambling. Psychological Science, 27(3), 299-311.

Pritchett, L. M., \& Murray, R. F. (2015). Classification images reveal decision variables and strategies in forced choice tasks. In Proceedings of the National Academy of Sciences (Vol. 112, Issue 23, pp. 7321-7326). https://doi.org/10.1073/pnas.1422169112

Raghunathan, R., \& Pham, M. T. (1999). All Negative Moods Are Not Equal: Motivational Influences of Anxiety and Sadness on Decision Making. In Organizational Behavior and Human Decision Processes (Vol. 79, Issue 1, pp. 56-77). https://doi.org/10.1006/obhd.1999.2838

Rouault, M., Seow, T., Gillan, C. M., \& Fleming, S. M. (2018). Psychiatric Symptom Dimensions Are Associated With Dissociable Shifts in Metacognition but Not Task Performance. In Biological Psychiatry (Vol. 84, Issue 6, pp. 443-451). https://doi.org/10.1016/j.biopsych.2017.12.017

Rubio, J. L., Ruiz-Veguilla, M., Hernández, L., Barrigón, M. L., Salcedo, M. D., Moreno, J. M., Gómez, E., Moritz, S., \& Ferrín, M. (2011). Jumping to conclusions in psychosis: a faulty appraisal. Schizophrenia Research, 133(1-3), 199-204.

Rutledge, R. B., Skandali, N., Dayan, P., \& Dolan, R. J. (2014). A computational and neural model of momentary subjective well-being. Proceedings of the National Academy of Sciences of the United States of America, 111(33), 12252-12257.

Sanders, J. I., Hangya, B., \& Kepecs, A. (2016). Signatures of a Statistical Computation in the Human Sense of Confidence. In Neuron (Vol. 90, Issue 3, pp. 499-506). https://doi.org/10.1016/j.neuron.2016.03.025 
Sano, A., Phillips, A. J., Yu, A. Z., McHill, A. W., Taylor, S., Jaques, N., Czeisler, C. A., Klerman, E. B., \& Picard, R. W. (2015). Recognizing academic performance, sleep quality, stress level, and mental health using personality traits, wearable sensors and mobile phones. 2015 IEEE 12th International Conference on Wearable and Implantable Body Sensor Networks (BSN), 1-6.

Schustek, P., Hyafil, A., \& Moreno-Bote, R. (2019). Human confidence judgments reflect reliability-based hierarchical integration of contextual information. In Nature Communications (Vol. 10, Issue 1). https://doi.org/10.1038/s41467-019-13472-z

Solovey, G., Shalom, D., Pérez-Schuster, V., \& Sigman, M. (2016). Perceptual learning effect on decision and confidence thresholds. Consciousness and Cognition, 45, 24-36.

Stone, A. A., Schneider, S., \& Harter, J. K. (2012). Day-of-week mood patterns in the United States: On the existence of "Blue Monday”, "Thank God it”s Friday' and weekend effects. In The Journal of Positive Psychology (Vol. 7, Issue 4, pp. 306-314). https://doi.org/10.1080/17439760.2012.691980

Taquet, M., Quoidbach, J., Fried, E. I., \& Goodwin, G. M. (2021). Mood Homeostasis before and during the Coronavirus Disease 2019 (COVID-19) Lockdown among Students in the Netherlands. In JAMA Psychiatry (Vol. 78, Issue 1, pp. 110-112). American Medical Association. https://doi.org/10.1001/jamapsychiatry.2020.2389

Taquet, M., Quoidbach, J., Gross, J. J., Saunders, K. E. A., \& Goodwin, G. M. (2020). Mood homeostasis, low mood, and history of depression in 2 large population samples. JAMA Psychiatry , 77(9), 944-951.

Taylor, S. A., Jaques, N., Nosakhare, E., Sano, A., \& Picard, R. (2017). Personalized Multitask Learning for Predicting Tomorrow's Mood, Stress, and Health. IEEE Transactions on Affective Computing, 1-1.

Torous, J., \& Firth, J. (2016). The digital placebo effect: mobile mental health meets clinical psychiatry. The Lancet. Psychiatry, 3(2), 100-102.

Triantafillou, S., Saeb, S., Lattie, E. G., Mohr, D. C., \& Kording, K. P. (2019). Relationship Between Sleep Quality and Mood: Ecological Momentary Assessment Study. JMIR Mental Health, 6(3), e12613.

Urai, A. E., Braun, A., \& Donner, T. H. (2017). Pupil-linked arousal is driven by decision uncertainty and alters serial choice bias. Nature Communications, 8(1), 1-11.

Van De Leemput, I. A., Wichers, M., Cramer, A. O. J., Borsboom, D., Tuerlinckx, F., Kuppens, P., Van Nes, E. H., Viechtbauer, W., Giltay, E. J., Aggen, S. H., Derom, C., Jacobs, N., Kendler, K. S., Van Der Maas, H. L. J., Neale, M. C., Peeters, F., Thiery, E., Zachar, P., \& Scheffer, M. (2014). Critical slowing down as early warning for the onset and termination of depression. Proceedings of the National Academy of Sciences of the United States of America, 111(1), 87-92.

Vickers, D. (1979). Confidence. In Decision Processes in Visual Perception (pp. 171-200). https://doi.org/10.1016/b978-0-12-721550-1.50011-9

Vickers, D. (2014). Decision Processes in Visual Perception. Academic Press. 
Vinckier, F., Rigoux, L., Oudiette, D., \& Pessiglione, M. (2018). Neuro-computational account of how mood fluctuations arise and affect decision making. Nature Communications, 9(1), 1708.

Wichers, M. (2014). The dynamic nature of depression: a new micro-level perspective of mental disorder that meets current challenges. In Psychological Medicine (Vol. 44, Issue 7, pp. 1349-1360). https://doi.org/10.1017/s0033291713001979

Wild-Hartmann, J. A. de, de Wild-Hartmann, J. A., Wichers, M., van Bemmel, A. L., Derom, C., Thiery, E., Jacobs, N., van Os, J., \& Simons, C. J. P. (2013). Day-to-day associations between subjective sleep and affect in regard to future depressionin a female population-based sample. In British Journal of Psychiatry (Vol. 202, Issue 6, pp. 407-412). https://doi.org/10.1192/bjp.bp.112.123794

Wyart, V., Nobre, A. C., \& Summerfield, C. (2012). Dissociable prior influences of signal probability and relevance on visual contrast sensitivity. Proceedings of the National Academy of Sciences of the United States of America. https://doi.org/10.1073/pnas.1120118109

Xu, H. (2020). Big Five Personality Traits and Ambiguity Management in Career Decision-Making. In The Career Development Quarterly (Vol. 68, Issue 2, pp. 158-172). https://doi.org/10.1002/cdq.12220

Xu, S., Liu, Q., \& Wang, C. (2021). Self-reported daily sleep quality modulates the impact of the framing effect on outcome evaluation in decision-making under uncertainty: An ERP study. In Neuropsychologia (Vol. 157, p. 107864). https://doi.org/10.1016/j.neuropsychologia.2021.107864

Yarkoni, T., \& Westfall, J. (2016). Bambi: A simple interface for fitting Bayesian mixed effects models. https://doi.org/10.31219/osf.io/rv7sn

Yeung, N., \& Summerfield, C. (2012). Metacognition in human decision-making: confidence and error monitoring. Philosophical Transactions of the Royal Society of London. Series B, Biological Sciences, 367(1594), $1310-1321$. 


\section{SUPPLEMENTARY MATERIAL}

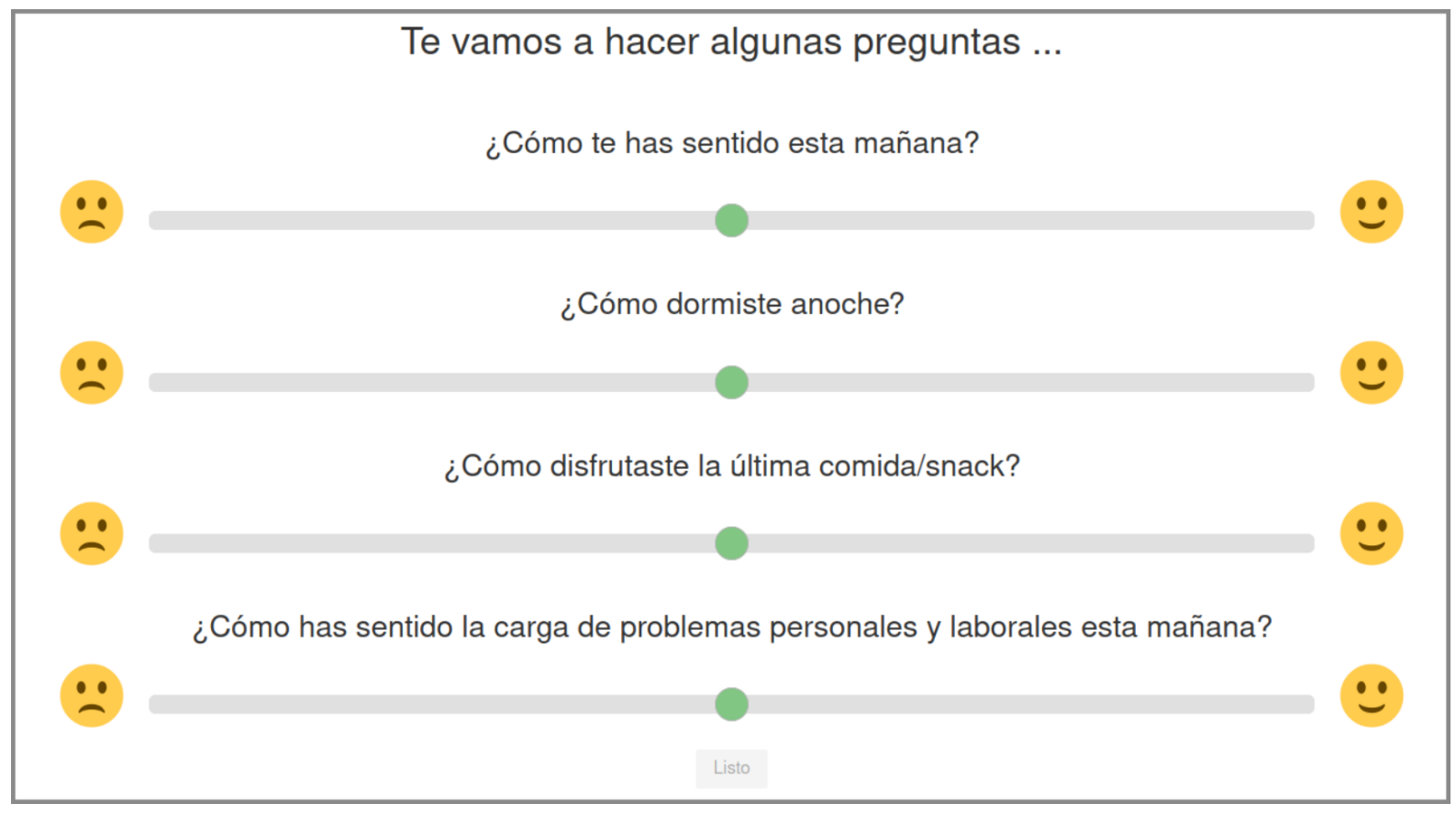

Supplementary figure 1. Quality of life self-reported questionnaire. Participants answered 3 or 4 questions about the quality of life states by moving cursors in continuous scales. In the morning sessions, they reported their mood ('How have you felt this morning?'), sleep quality ('How did you sleep last night?'), food enjoyment ('How did you enjoy your last meal/snack?'), and stress level ('How did you feel about your personal and working problems this morning?'). The same questions were asked in the afternoon session changing the word 'mañana' (morning) for 'tarde' (afternoon), except for the sleep question which was skipped.
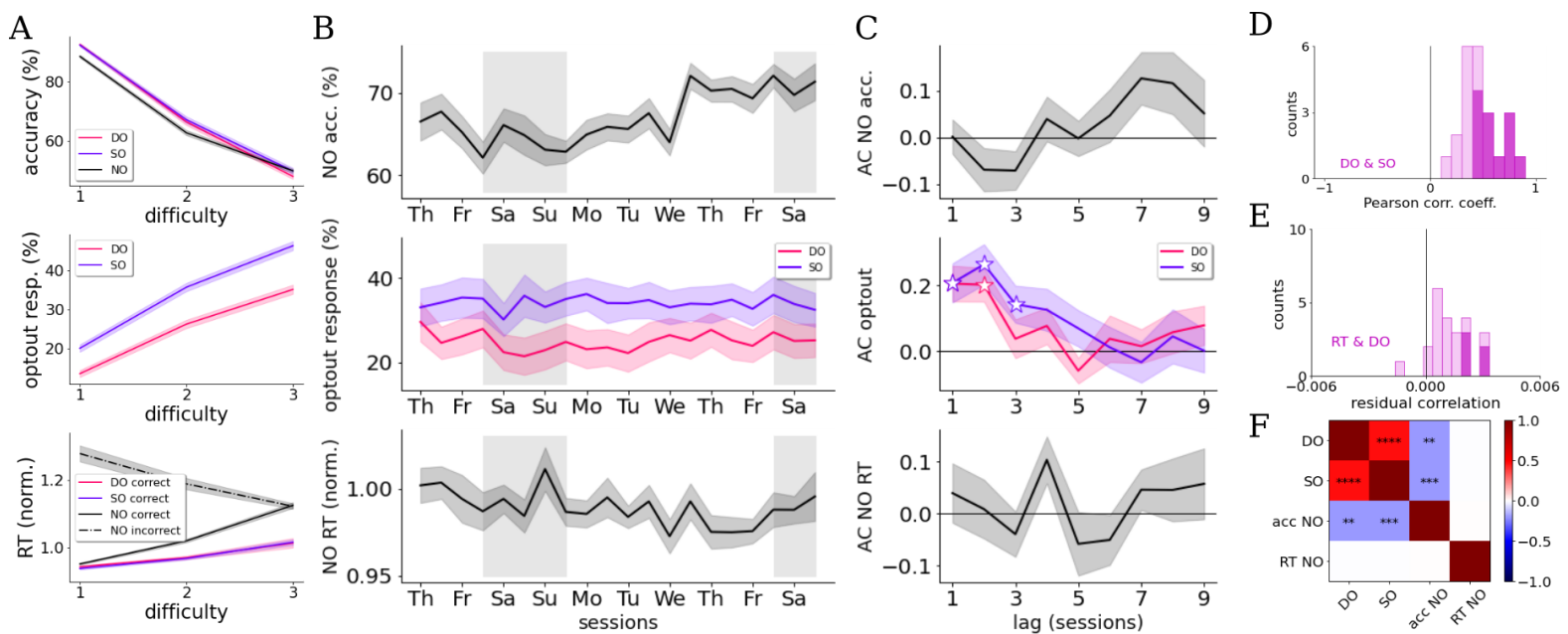

Supplementary figure 2. Psychometric variables fluctuations (Orientation task): A. Mean values across participants and sessions vs. stimulus difficulty. Shaded areas indicate standard error of the mean (s.e.m.). Top panel: accuracy percentage in the three types of trials. Middle panel: percentage of opt-out of each stage. Bottom panel: reaction time over 
correct trials and incorrect non-optout trials. B. Mean (line) and s.e.m. (shaded areas) values across participants. Gray shaded area indicating weekend sessions. C. Autocorrelation coefficients in non-optout accuracy, opt-out selection and reaction time of correct non-optout trials (star: $p<0.05$ after correction). D. Distribution of Pearson across-session correlation coefficients of the proportion of deterministic (DO) and stochastic (SO) opt-outs. Filled bars indicate subjects with significant correlation $(p<0.05)$ at individual level. E. Linear mixed model residual correlation between reaction time in correct non-optout trials and the proportion of the deterministic opt-out response $\left(n=n_{\text {sessions }} \times n_{\text {difficulties }}=20 \times 3\right)$. Filled bars indicate subjects with significant correlation $(p<0.05)$ at individual level. F. Matrix representing the mean correlation coefficient across participants $\left({ }^{* *} p<0.01,{ }^{* * *} p<0.001\right.$, ${ }^{* * * *} p<10^{-4}$ uncorrected).

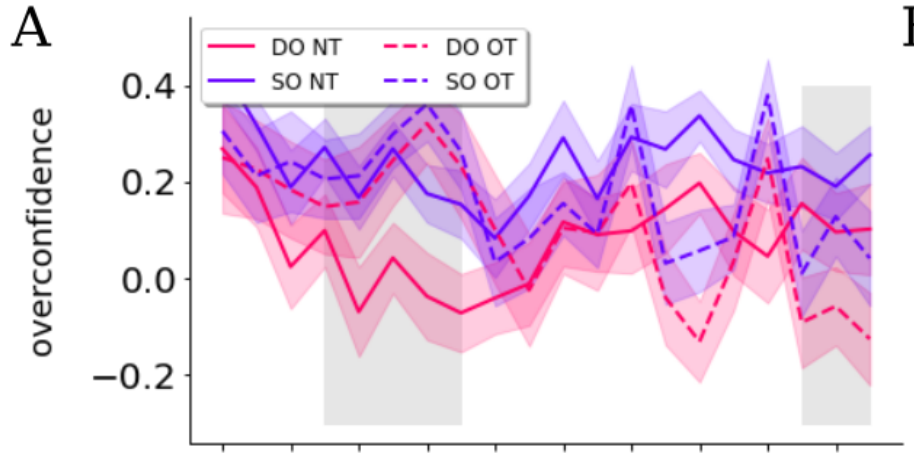

Th Fr Sa Su Mo Tu We Th Fr Sa

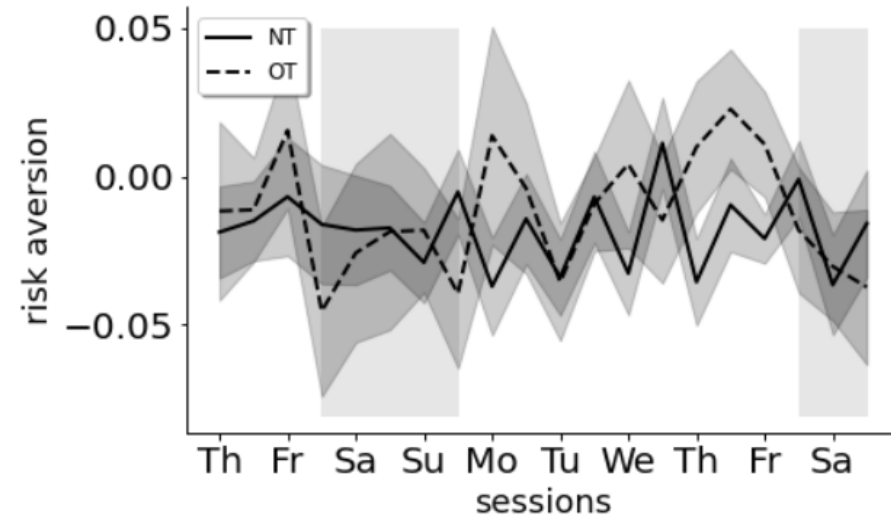

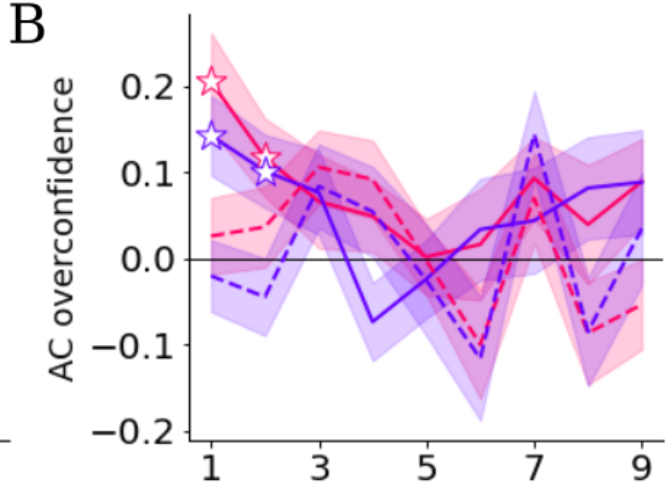

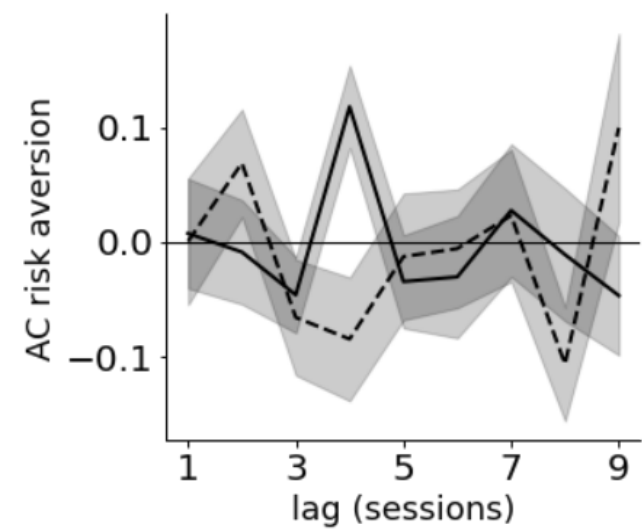

Supplementary figure 3. Overconfidence \& risk aversion fluctuations: A. Mean (line) and s.e.m. (shaded areas) values across participants. Gray shaded area indicating weekend sessions. B. Autocorrelation coefficients (star: $p<0.05$ after correction).

\begin{tabular}{|l|l|l|l|l|}
\hline ACC lag 1 & NT (t) & NT (p) & OT (t) & OT (p) \\
\hline mood & 2.83 & 0.008 & 0.01 & 0.99 \\
\hline food & 1.00 & 0.3 & 0.37 & 0.7 \\
\hline stress & 1.45 & 0.02 & 1.13 & 0.2 \\
\hline
\end{tabular}

Supplementary Table 1. Difference in auto-correlation of mood state reports (mood, food enjoyment and stress level) at lag 1, between for morning-to-afternoon and 
afternoon-to-morning correlation impact in. Numbers represent the t-statistic and p-value for two-paired t-test for both cohorts: numerosity (NT) and orientation (OT) task groups.

\begin{tabular}{|l|l|l|l|l|l|}
\hline \multicolumn{2}{|c|}{} & \multicolumn{2}{l|}{ Numerosity task } & \multicolumn{2}{l|}{ Orientation task } \\
\cline { 3 - 6 } \multicolumn{2}{|c|}{} & Intercept & report & Intercept & report \\
\hline \multirow{7}{*}{ optout } & mood & 25.3 & 1.34 & 26.52 & -2.17 \\
\cline { 2 - 6 } & food & 23.03 & 4.1 & 24 & 1.62 \\
\cline { 2 - 6 } & sleep & 27.9 & -0.85 & 28.01 & -4.2 \\
\cline { 2 - 6 } & stress & 25.35 & 1.76 & 24 & 2.97 \\
\hline \multirow{2}{*}{ RT } & mood & 1 & -0.03 & 0.98 & 0.01 \\
\cline { 2 - 6 } & food & 0.98 & 0.01 & 1 & 0.02 \\
\cline { 2 - 6 } & sleep & 0.99 & 0 & 0.98 & 0.01 \\
\hline & stress & 0.97 & 0.03 & 0.99 & 0.01 \\
\hline
\end{tabular}

Supplementary Table 2. Quality of life effect on self-confidence. Fixed effects weights in the Bayesian linear mixed model. The different lines correspond to particular choices for the confidence proxy (either the average proportion of optouts or the median reaction time in correct NO trials in the session) and for the self-report. 

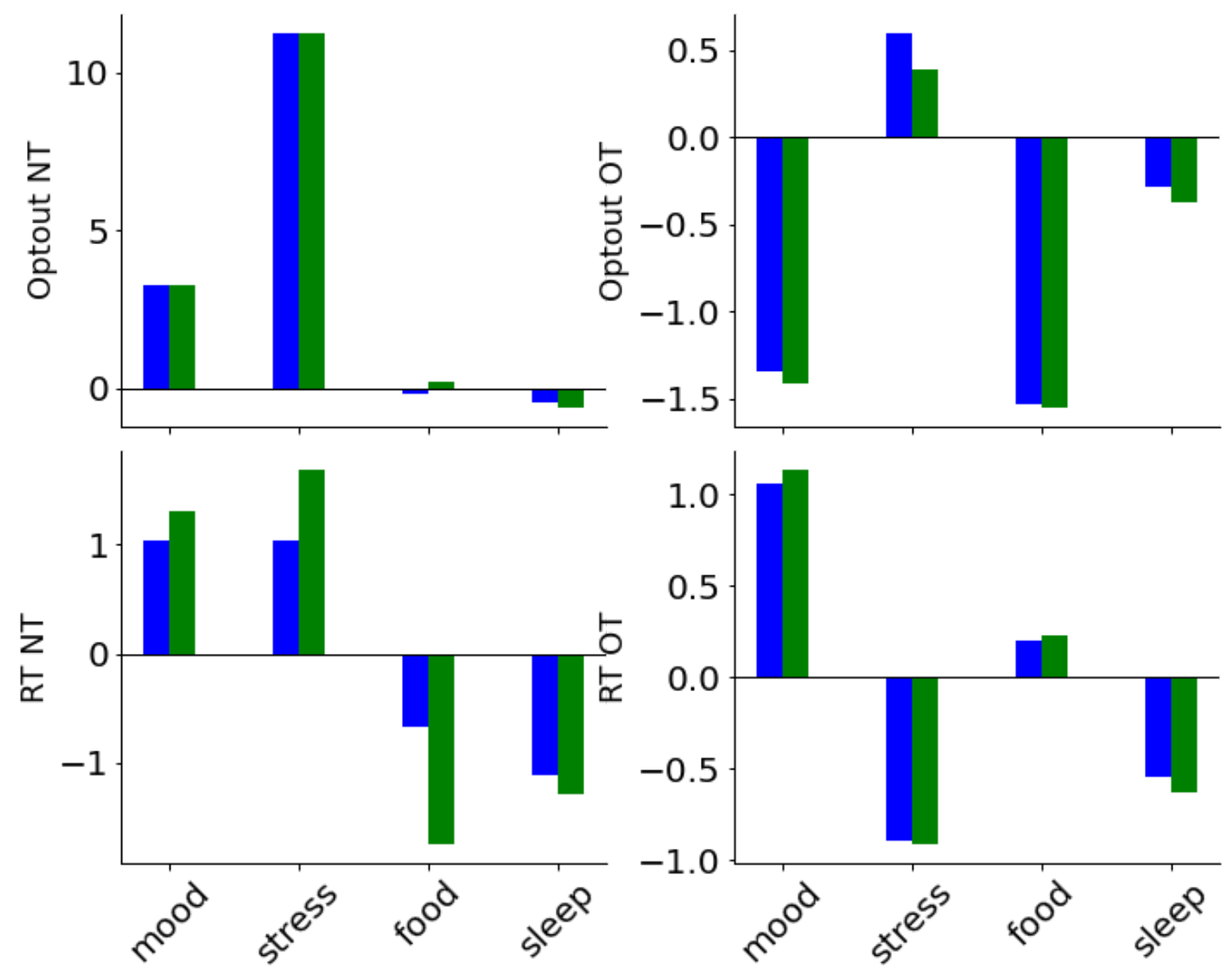

Supplementary Figure 4. Model comparison for BLMM quality of life effect on self-confidence. WAIC (Watanabe-Akaike Information Criterion) in blue bars and LOO-cV (Leave-One-Out cross-validation criterion) in green bars of the model $(\sim 1+1$ |participant + report + report|participant) minus the corresponding WAIC and LOO-cV of the control model $(\sim 1+1$ participant). A positive value indicates that the model accounts better for the data than the control model. 

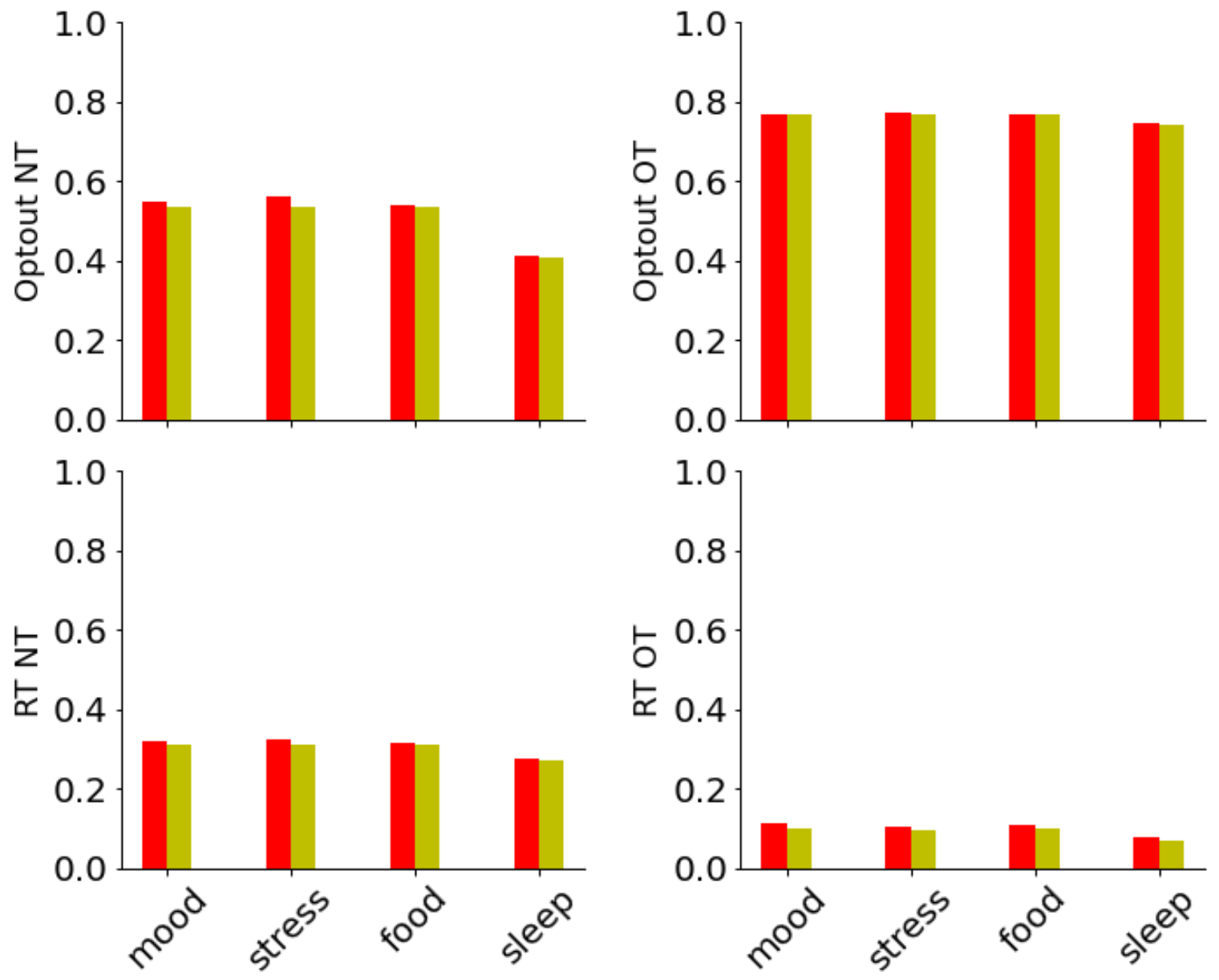

Supplementary Figure 5. R-squared value for BLMM quality of life effect on self-confidence. R-squared value for the tested model $(\sim 1+1$ participant + report + report|participant) in red bars and $\mathrm{R}$-squared value for the control model $(\sim 1+1$ |participant $)$ in light brown bars. 